\title{
Oligodendrocyte precursor cells prune axons in the mouse neocortex
}

JoAnn Buchanan ( $\square$ joannb@alleninstitute.org )

Allen Institute for Brain Sciences

\section{Leila Elabbady}

Allen Institute for Brain Science

\section{Forrest Collman}

Allen Institute for Brain Science

Nicholas Jorstad

Allen Institute for Brain Science

Trygve Bakken

Allen Institute for Brain Science https://orcid.org/0000-0003-3373-7386

\section{Carolyn Ott}

Janelia Research Campus HHMi

Jenna Glazer

Johns Hopkins University School of Medicine

\section{Adam Bleckert}

Allen institute for Brain Science

Agnes Bodor

Allen Institute for Brain Science

Derrick Brittain

Allen Institute for Brain Science

\section{Dan Bumbarger}

Allen Institute

\section{Gayathri Mahalingam}

Allen Institute for Brain Science

\section{Sharmishtaa Seshamani}

Allen Institute for Brain Science

\section{Casey Schneider-Mizell}

Allen Institute for Brain Science

\section{Marc Takeno}

Allen Institute for Brain Science https://orcid.org/0000-0002-8384-7500

\section{Russel Torres}

Allen Institute for Brain Science https://orcid.org/0000-0002-2876-4382 
Allen institute for Brain Science

\section{Rebecca Hodge}

Allen Institute for Brain Science https://orcid.org/0000-0002-5784-9668

\section{Manuel Castro}

Princeton University https://orcid.org/0000-0003-2787-0332

\section{Sven Dorkenwald}

Princeton University

\section{Dodam Ih}

\section{Princeton University}

\section{Chris Jordan}

WiredDifferently, Inc.

\section{Nico Kemnitz}

Princeton University

\section{Kisuk Lee}

Massachusetts Institute of Technology

\section{Ran Lu}

Princeton University

\section{Thomas Macrina}

Princeton University https://orcid.org/0000-0002-0622-9338

\section{Shang Mu}

Princeton University

\section{Sergiy Popovych}

Princeton University

William Silversmith

Princeton University

Ignacio Tartavull

Princeton University

Nicholas Turner

Princeton University

\section{Alyssa Wilson}

Princeton University

\section{William Wong}

Princeton University

\section{Jingpeng $\mathbf{W u}$}

Princeton University

\section{Aleksandar Zlateski}

Princeton University

Jonathan Zung

Princeton University 


\section{Jennifer Lippincott-Schwartz}

Janelia Research Campus, Howard Hughes Medical Institute, Ashburn, VA, 20147

https://orcid.org/0000-0002-8601-3501

\section{Ed S. Lein}

Allen Institute for Brain Science https://orcid.org/0000-0001-9012-6552

\section{H. Sebastian Seung}

Princeton University https://orcid.org/0000-0002-8591-6733

\section{Dwight Bergles}

Johns Hopkins University https://orcid.org/0000-0002-7133-7378

\section{R. Clay Reid}

Allen Institute for Brain Science https://orcid.org/0000-0002-8697-6797

\section{Nuno da Costa}

Allen Institute for Brain Science

\section{Biological Sciences - Article}

Keywords: serial electron microscopy, glial types, visual cortex, oligodendrocyte precursor cells

Posted Date: June 29th, 2021

DOI: https://doi.org/10.21203/rs.3.rs-581121/v1

License: (c) (i) This work is licensed under a Creative Commons Attribution 4.0 International License. Read Full License 


\section{Oligodendrocyte precursor cells prune axons in the mouse neocortex}

JoAnn Buchanan ${ }^{1,7^{*}}$, Leila Elabbady ${ }^{1}$, Forrest Collman ${ }^{1}$, Nikolas L. Jorstad ${ }^{1}$,Trygve E.

4 Bakken ${ }^{1}$, Carolyn Ott ${ }^{2}$, Jenna Glatzer ${ }^{3}$, Adam A. Bleckert ${ }^{1}$, Agnes L. Bodor ${ }^{1}$, Derrick Brittan ${ }^{1}$,

5 Daniel J. Bumbarger ${ }^{1}$, Gayathri Mahalingam ${ }^{1}$, Sharmishtaa Seshamani ${ }^{1}$, Casey Schneider-

6 Mizell ${ }^{1}$, Marc M. Takeno ${ }^{1}$, Russel Torres ${ }^{1}$, Wenjing Yin ${ }^{1}$, Rebecca D. Hodge ${ }^{1}$, Manuel Castro ${ }^{4}$,

7 Sven Dorkenwald ${ }^{4,5}$, Dodam $\mathrm{Ih}^{4}$, Chris S. Jordan ${ }^{4}$, Nico Kemnitz ${ }^{4}$, Kisuk Lee, ${ }^{4,5}$, Ran $\mathrm{Lu}^{4}$,

8 Thomas Macrina ${ }^{4}$, Shang $\mathrm{Mu}^{4}$, Sergiy Popovych ${ }^{5}$, William M. Silversmith ${ }^{5}$, Ignacio Tartavull ${ }^{5}$,

9 Nicholas L. Turner ${ }^{4,5}$ Alyssa M. Wilson ${ }^{4}$, William Wong ${ }^{4}$, Jingpeng $\mathrm{Wu}^{4}$, Aleksandar Zlateski ${ }^{4}$,

10 Jonathan Zung ${ }^{4}$, Jennifer Lippincott-Schwartz ${ }^{2}$, Ed S.Lein ${ }^{1}$, H. Sebastian Seung ${ }^{4,5}$ Dwight E.

11 Bergles ${ }^{3,6}$, R. Clay Reid ${ }^{1}$, Nuno Maçarico da Costa $^{1,7^{*}}$

${ }^{1}$ Allen Institute for Brain Sciences

${ }^{2}$ Janelia Research Campus, Howard Hughes Medical Institute

${ }^{3}$ The Solomon H. Snyder Department of Neuroscience, John Hopkins University School of Medicine

${ }^{4}$ Princeton Neuroscience Institute, Princeton University

${ }^{5}$ Computer Science Department, Princeton University

${ }^{6}$ Johns Hopkins Kavli Neuroscience Discovery Institute

${ }^{7}$ Corresponding Authors 


\section{ABSTRACT}

31 Neurons in the developing brain undergo extensive structural refinement as nascent circuits adopt

32 their mature form ${ }^{1}$. This transformation is facilitated by the engulfment and degradation of excess

33 axonal branches and inappropriate synapses by surrounding glial cells, including microglia and

34 astrocytes $^{2,3}$. However, the small size of phagocytic organelles and the complex, highly ramified morphology of glia has made it difficult to determine the contribution of these and other glial cell types to this process. Here, we used large scale, serial electron microscopy (ssEM) with computational volume segmentation to reconstruct the complete 3D morphologies of distinct glial types in the mouse visual cortex. Unexpectedly, we discovered that the fine processes of oligodendrocyte precursor cells (OPCs), a population of abundant, highly dynamic glial progenitors $^{4}$, frequently surrounded terminal axon branches and included numerous phagolysosomes (PLs) containing fragments of axons and presynaptic terminals. Single- nucleus RNA sequencing indicated that cortical OPCs express key phagocytic genes, as well as neuronal transcripts, consistent with active axonal engulfment. PLs were ten times more abundant in OPCs than in microglia in P36 mice, and declined with age and lineage progression, suggesting that OPCs contribute very substantially to refinement of neuronal circuits during later phases of cortical development.

\section{MAIN}

OPCs emerge from several germinal zones in late prenatal development following the sequential generation of neurons and astrocytes, migrate into the expanding cortex and then proliferate to establish a grid-like distribution, with individual cells occupying distinct territories. Genetic fate tracing and time lapse imaging in vivo have demonstrated that these progenitors play a critical role in generating oligodendrocytes and thus myelin throughout the central nervous system (CNS) ${ }^{5}$. However, OPCs are present in some cortical regions weeks before oligodendrogenesis begins and extend highly ramified processes that developing neurons. These progenitors express a diverse set

56 of neurotransmitter receptors and form direct, functional synapses with excitatory and inhibitory 57 neurons. ${ }^{6,7}$.These features have traditionally been viewed through the perspective of 58 oligodendrogenesis ${ }^{8}$, as other functions for these ubiquitous glial cells have not been clearly 59 established. Our knowledge about the structure and function of OPCs has been severely limited by 60 incomplete ultrastructural information, because their fine, highly branched processes are difficult 
61 to unambiguously identify in EM studies without complete reconstructions to connect them to 62 identifiable somata. Modern computational volume EM methods offer new opportunities to

63 increase our understanding of brain ultrastructure ${ }^{9-12}$, particularly for morphologically complex,

64 highly dynamic glial cells like OPCs. Here, we used two densely segmented and reconstructed 65 datasets of mouse visual cortex, ages P36 (Fig. 1a) and P49, to perform a detailed morphometric analysis and quantification of the anatomical features of somata, processes, and organelles of OPCs

67 at this highly dynamic phase of neocortical maturation to help define their roles in the developing 68 brain.

\section{Structural features of OPCs revealed by large scale serial EM}

71 Past EM ultrastructural studies defined several common morphological characteristics of OPCs, such as their bean-shaped nucleus containing low heterochromatin and presence of centrioles in their cytoplasm ${ }^{13}$, consistent with their proliferative progenitor state. ${ }^{14}$. Originally referred to as a multipotential type of glia ${ }^{4}$, and often described as NG2 cells ${ }^{15,16}$ in reference to their expression of the proteoglycan NG2, early EM investigations often focused on their cellular responses to injury and the close association of their processes with synapses and degenerating nerve fibres ${ }^{15,17}$. More recent studies indicate that OPC processes form direct synapses with axons and that OPCs contact nodes of Ranvier ${ }^{6,18}$ in naïve animals; however, quantitative analysis of these structural features has been difficult due to the limited sampling $6,15,17,18$.

The two EM volumes P36 (Fig. 1.a) and P49 we examined in serial sections contained four distinct classes of glia: OPCs (Fig. 1b, c), microglia (Fig. 1d), oligodendrocytes (Fig.1e), and astrocytes (Fig. 1f). OPCs exhibited a ramified form with 15 to 17 highly branched processes extending up to $50 \mu \mathrm{m}$ radially from the soma (Fig. 1b, c, g, h, Extended Data Fig.1 c-j) that contained numerous filopodia on their tips. 3D renderings of multiple cells revealed that OPCs, similar to astrocytes and microglia ${ }^{19,20}$, formed a grid-like organization with little overlap between territories of 87 adjacent cells of the same class (Fig. 1b), and also contacted synapses ${ }^{21,22}$. The OPC somata were frequently in a satellite position, like those of microglia and oligodendrocytes ${ }^{23,24}$ (Fig. 1g, Extended Data Fig. 1c-k;Video 1), and were remarkably variable in both size and shape, ranging

90 from elongated or bean shaped to smooth or irregular with a rough surface (Fig. 1b; Extended Data

91 Fig. 1c-j). OPCs were readily distinguished from other glial cell types by these features as well as 
92 having: 1) larger nuclei that were elongated and contained less heterochromatin than those of 93 microglia (Fig. 1i-k); 2) processes that were longer and smoother compared to microglia or 94 astrocytes (Fig. 1c, d, f); 3) axons that formed synaptic contacts with their processes (Fig. 11, m);

95 4) cytoplasm that was more electron lucent than that of microglia and devoid of glycogen granules 96 that are numerous in astrocytes (Fig. 1m); and 5) the presence of primary cilia, which were not 97 found in microglia, pre-myelinating or mature oligodendrocytes (Fig. 1n,o; Extended Data Fig. $98 \quad 1 \mathrm{c}-\mathrm{j}, 1 \mathrm{k})$.

100 OPC processes contain numerous lysosomes and phagolysosomes

101 Previous in vivo imaging experiments revealed the dynamic nature of OPC processes, which 102 exhibit continuous branch remodeling as they migrate through the gray matter, making transient 103 interactions with various cellular constituents ${ }^{25}$. Based on profiles in EM images and associated 104 cellular reconstructions, we find that OPC processes were often found in contact with axons 105 (Fig.2a-d). Unexpectedly, the cytoplasm of these processes often contained numerous membrane106 bound organelles that included phagosomes and primary and secondary lysosomes, termed 107 phagolysosomes(PLs)(Fig. 2b,c,e), suggesting that OPCs at this age are engaging in phagocytosis. 108 The process of phagocytosis begins with recognition of a target, followed by phagosome formation 109 (engulfment) and maturation, and then fusion of the phagosome with a lysosome, forming a PL 26. 110 In both EM volumes, phagosomes were identified as completely internalized, membrane delimited 111 structures. Lysosomes appeared electron dense and measured $\sim 500 \mathrm{~nm}$ in diameter (Fig. 2b), while 112 PLs were multichambered, large organelles measuring $750 \mathrm{~nm}$ (Fig. 2e-n). Seldom visualized in 113 large numbers by EM, PLs represents the final step of phagocytosis, and constitute a highly acidic 114 compartment capable of destroying ingested elements ${ }^{26,27}$. Mapping the distribution of PLs in one 115 OPC (Fig. 2o) demonstrated that they were widely distributed within the cell, but particularly 116 prevalent near the tips of OPC processes. This finding led us to count PLs in eight more OPCs in 117 P36 dataset for comparison.

119 PLs in OPCs were large enough to visualize their internal contents. Although much of this material 120 was unidentifiable cellular debris, they frequently contained clusters of small $(\sim 40 \mathrm{~nm})$, clear 121 vesicles (Fig. 2 f,i-n), suggesting that OPCs had engulfed portions of presynaptic terminals. 122 Occasionally, there was evidence of an entire synaptic terminal surrounded by OPC cytoplasm, 
123 with both presynaptic vesicles and a post-synaptic density visible (Fig. 2 f,g). Notably, fragments

124 of myelin, distinct organelles (e.g. mitochondria), and cytoskeletal elements, were not observed in

125 these PLs, suggesting that OPCs primarily engulf axonal processes. In contrast, although

126 microglial PLs also contained unidentifiable electron dense material, they did not contain $40 \mathrm{~nm}$

127 vesicles (Extended Data Fig. 2b-e, h), suggesting that, at this age, these two glial cell types

128 recognize and remove distinct cellular elements. The presence of abundant PLs in OPCs containing

129 synaptic material was unexpected, as most structural pruning of neurons is thought to be mediated

130 by microglia ${ }^{2,28}$. To determine the relative abundance of PLs in these two glial cell types, we

131 quantified PLs in complete 3D reconstructions of microglia in the same volumes. This analysis

132 revealed that PLs were significantly more abundant in OPCs than microglia at this age (Fig.

133 2o,p;Extended Data Fig. 2) (Total PLs/cell: OPCs, $108 \pm 48$; microglia, $9 \pm$ 3; student's t-test, p <

$1340.001, \mathrm{n}=9$ ); although OPCs are larger than microglia, the density of PLs was still significantly

135 higher in OPCs (Fig. 2q; Extended data Fig. 2f,g) (PL density: OPCs, $0.143 \pm 0.05$ PLs/ $\mu \mathrm{m}^{3}$;

136 microglia, $0.03 \pm 0.01 \mathrm{PLs} / \mu \mathrm{m}^{3} ; \mathrm{p}<0.001, \mathrm{n}=9$ ), suggesting that OPCs are more actively engaged

137 in neuronal process engulfment at this age.

139 To further explore the prevalence of these organelles in OPCs, OPCs isolated from the visual 140 cortex of one month-old mice were immunolabeled for lysosomal associated membrane protein 2 141 (LAMP-2), which is associated with both lysosomes and phagolysosomes ${ }^{29}$, and NG2 (chondroitin

142 sulfate proteoglycan 4) that specifically labels $\mathrm{OPCs}^{15}$. Consistent with the ultrastructural 143 observations described above, OPCs at this age contained 25 to 54 LAMP-2 immunoreactive 144 circular organelles distributed throughout their somata and processes (Extended Data Fig.1a,b), 145 indicating a high investment in cellular turnover in the developing cortex.

147 During the critical period of mouse visual cortex from P21 to P35, microglia engulf and eliminate 148 synapses $^{30,31}$ and neuronal corpses through phagocytosis and eventual digestion via acidified 149 phagosomes and PLs s ${ }^{32}$. This structural remodeling of cortical connections declines rapidly after 150 the first postnatal month ${ }^{2,33}$, coinciding with the end of the critical period of ocular dominance 151 plasticity in the visual cortex. To determine if PLs in OPCs follow a similar developmental 152 progression, we quantified PLs in OPCs reconstructed from serial EM volumes obtained in the 153 P49 mouse visual cortex. Significantly fewer PLs were present in OPCs at this age, despite the 
154 similar volume occupied by these cells (Extended Data Fig. 3e, f, g) (PL density: P36 OPCs, 0.143

$155 \pm 0.05 \mathrm{PLs} / \mu_{\mathrm{m}}^{3} ;$ P49 OPCs, $0.085 \pm 0.03 \mathrm{PLs} / \mu \mathrm{m}^{3} ; \mathrm{p}=0.01$, student's $\mathrm{t}$-test, $\mathrm{n}=9$, 11,

156 respectively). Together, these results indicate there is a temporal shift in OPC behavior over time

157 and that OPCs may contribute to an early phase of structural remodeling of neurons during the

158 final phase of the critical period in the visual cortex.

160 OPCs undergo dramatic changes in gene expression and morphology as they differentiate into 161 myelin forming oligodendrocytes ${ }^{8}$ (Extended Data Fig. 3a-d). To determine if axonal engulfment 162 is preferentially associated with the OPC progenitor state, we also examined the cytosol of 163 premyelinating and mature oligodendrocytes at P49 (due to the smaller volume, there was only 164 one partially reconstructed premyelinating oligodendrocyte in the P36 dataset). These more mature 165 oligodendroglia were distinguished from OPCs by the lack of primary cilia ${ }^{34}$, as well as the 166 presence of sheets of membrane extending along axons and membrane wraps indicative of nascent 167 myelination (Extended Data Fig. 3a-d). Premyelinating oligodendrocytes had lower densities of 168 PLs than OPCs PL density: P49 OPCs, $0.085 \pm 0.03$ PLs/ $\mu m^{3}$; P49 Pre-myelinating 169 oligodendrocyte, $0.034 \pm 0.018 \mathrm{PLs} / \mu \mathrm{m}^{3} ; \quad \mathrm{p}=0.001$, student's $\mathrm{t}$-test, $\mathrm{n}=11,5$, 170 respectively,(Extended Data Fig. 3f). In fully mature oligodendrocytes, PLs were extremely rare 171 (extended figure 3.e), suggesting that engulfment of neuronal processes declines rapidly as OPCs 172 undergo differentiation (Extended Data, Fig. 3e, f, g).

\section{OPCs express genes that enable phagocytosis}

175 The 3D EM reconstructions indicate that OPC processes are filled with numerous PLs that contain 176 neuronal material, suggesting that they assist in remodeling nascent neuronal circuits through 177 engulfment of axons and synapses. Phagocytosis requires a complex array of proteins to recognize 178 and engulf distinct cargos, the components for which are highly conserved among different 179 species. To determine whether OPCs express genes that would enable this behavior, we analyzed 180 existing single nucleus RNA-seq and DNA-methylation data sets derived from the mouse motor 181 cortex (P56) ${ }^{35}$ for both lysosomal and phagocytic genes (Fig. 3a,b). OPC nuclei were identified by 182 co-expression of pdgfra and olig2 and premyelinating/myelinating oligodendrocytes were 183 identified by expression of $\operatorname{opalin}($ Fig.3 a-c). Expression of genes that encode components of 184 phagocytosis were then compared between OPCs, microglia and mature oligodendrocytes 
185 (Fig.3a,b), identified by previous characterization ${ }^{35}$ and expression of distinct marker genes 186 (Extended Data Figure 4).

188 Both OPCs and microglia expressed high levels of mRNAs encoding phagocytic and lysosomal genes. Of the 38 phagocytosis-related genes examined, 32\% were expressed in OPCs and $89 \%$ were expressed by microglia (Fig.3b). Eight phagocytic genes were expressed in both glial types,

191 including Mertk, Ptprj, and Lrp1. Mertk (Mer tyrosine kinase) is a member of the TAM (Tyro3-

192 Axl-Mer) family of receptors that signal engulfment, which astrocytes use to refine connectivity

193 in the adult mouse hippocampus by phagocytosing excitatory synapses ${ }^{36}$. Ptprj is a tyrosine

194 phosphatase receptor protein that is known to regulate phagocytosis and migration in microglia ${ }^{37}$,

195 and Lrpl is a low density lipoprotein receptor essential for myelin phagocytosis and regulation of

196 inflammation in OPCs ${ }^{38}$. Several genes that encode phagocytosis-related proteins expressed in

197 OPCs were absent from microglia: Raplgap, a GTPase activating protein that mediates FcyR

198 dependent phagocytosis ${ }^{39}$; and Xrkr4 and Xrkr6, proteins that promote the exposure of 199 phosphatidylserine to produce "eat me" signaling during phagocytosis, which are highly expressed 200 in the developing brain ${ }^{40}$. The distinct complement of phagocytic genes expressed by OPCs and 201 microglia may enable these cells to identify and engulf discrete parts of neurons. Notably, 202 expression of all the phagocytosis-related genes were negligeable in oligodendrocytes, consistent 203 with the much higher incidence of PLs in OPCs.

205 We also searched for the presence of neuronal transcripts within microglia and OPCs that may 206 have been engulfed along with neuronal processes. Neuronal transcripts were found in both OPCs 207 and microglia, which suggests both types of glia are phagocytosing neurons at this age Fig.3c,d). 208 However, transcripts encoding these components were higher in OPCs, which correlates with the 209 increased number of PLs in these cells. Because OPCs express many proteins characteristic of 210 neurons, such as AMPA and NMDA glutamate receptors and voltage-dependent $\mathrm{Na}^{+}$channels, we 211 assessed the likelihood that transcription of traditionally "neuronal" genes is occurring directly in 212 OPCs by examining the methylation state of the neuronal genes; genes that are actively transcribed 213 should be hypomethylated. This analysis revealed that microglia and OPCs lack hypomethylation 214 at most neuronal genes (Fig. 3e), a sign that they do not express these genes but incorporated them 215 from elsewhere, likely by phagocytosis of neuronal material. Analysis of cell specific gene 
216 expression data suggest that OPCs ingest material from diverse neurons, rather than targeting a

217 specific subtype. These findings, together with the ultrastructural data, provide further evidence

218 that OPCs phagocytose axons of many types of neurons in the mammalian cortex.

220 OPCs prune axons in the mouse cortex

221 The appearance of clusters of $40 \mathrm{~nm}$ clear vesicles inside OPC PLs suggests that presynaptic 222 terminals or portions of axons containing terminals are engulfed by these cells. If this process is 223 frequent, OPCs engaged in various stages of this process should be visible in the EM datasets. 224 Indeed, 3D reconstructions revealed that terminal branches of axon collaterals were often 225 surrounded by OPC processes (Fig. 4a-f). The complex anatomy of the branches and the need to 226 accurately manually annotate and quantify the ingestions, made this analysis particularly 227 challenging. Therefore, two individual main branches from two distinct OPCs in the P36 dataset 228 were examined in detail (OPCs \#1, \#7) (Extended Data Fig. 1c,k; Extended data Fig. 6a-h). We 229 categorized the ingestions as phagosomes (PS), PLs or axon engulfment (Fig. 4g). For the latter, 230 there was no evidence of injury or destruction to the axon, only local contact with OPC cytoplasmic 231 processes that encased the collateral or terminal region of the axon branch. Two types of axon 232 engulfment were observed: either small axonal pieces less than $1 \mu \mathrm{m}$ in length were surrounded 233 by an OPC process, in which the collateral or terminal axon branch remained connected to the 234 parent axon (24 of 37 in OPC \#1, 23 of 32 in OPC \#7), or larger axonal collateral or terminal 235 branch segments (up to $3 \mu \mathrm{m}$ ) were ensheathed (9 of 37, OPC \#1 and 3 of 32 OPC \#7), but remained 236 attached to the parent axon (Fig. 4b,e; Extended Data Fig. 4a-h; Video 3).

OPCs form synapses with both excitatory and inhibitory neurons in the developing cortex ${ }^{6,7}$ and axons from both neuron classes are targeted for myelination in the cortex ${ }^{41,42}$, indicating that OPCs and their subsequent later developmental stages interact with distinct cell types. To determine if axon pruning is specific to one class of neurons, we traced the branches of 70 axons ensheathed

242 by OPCs back to the main axon and examined the boutons, classifying them as excitatory or 243 inhibitory based on synaptic morphology. This analysis showed the predominant type of axons 244 within the two isolated branches of OPCs were excitatory (89\% in OPC \#1 and 81\% in OPC \#7 245 of ensheathed structures) (Fig. 4h,i) with 8\% undetermined. Approximately $3 \%$ of the engulfed axons were identified as inhibitory, suggesting that OPCs were mainly removing portions of 
247 excitatory axons, which comprise the majority of axons in cortical gray matter. This number may

248 be lower than the 10-20\% inhibitory neuron population in the neocortex $\left({ }^{43}\right)$, because the sample

249 size was limited to layer $2 / 3$ and only 2 OPC branches. Furthermore, the transcriptomics data

250 suggests that OPCs are non-discriminatory phagocytes and engulf all neuron types, based on all

251 layers of the cortex. The association of OPCs with terminal and collateral axon branches was

252 observed in all OPCs from both P36 and P49 datasets, indicating that axon pruning is a conserved

253 function of OPCs during this period. Such associations may represent the predecessor to axonal

254 engulfment and suggest that the terminal portion of axon collaterals are targeted for removal by

255 OPCs.

256

\section{Discussion}

258 Consolidation of nascent networks into stable, but adaptable, circuits requires extensive 259 remodeling during development, when excess neurons, axon collaterals and even individual 260 synapses are removed ${ }^{1,44,45}$. This structural refinement is aided by glia, which recognize and engulf 261 both cellular debris and portions of intact neurons ${ }^{46,47}$. Microglia are key participants in this 262 process, as they express phagocytic genes (e.g. Merktk, Dock2, Sirpa) ${ }^{48}$, engage in constant 263 surveillance through process motility, can transform into amoeboid, highly phagocytic cells, that 264 accumulate presynaptic material in their cytoplasm ${ }^{31}$. Astrocytes also express phagocytic 265 components (e.g. Mertk, Megf10) ${ }^{3}$ and participate in excitatory synapse removal in adult mice ${ }^{49}$, 266 indicating that structural refinement of neurons is the shared responsibility of multiple glial cell 267 types.

269 This new analysis of the 3D ultrastructure of individual glial cells in mouse visual cortex using 270 volumetric EM, provides strong evidence that OPCs also participate in neuronal circuit refinement. 271 OPCs, a widely distributed, and abundant population of lineage restricted progenitor cells, 272 extended fine processes that made numerous contacts with terminal axon branches and synapses 273 of excitatory neurons, surrounding and enveloping neuronal components within cup shaped and 274 sheetlike cytoplasmic formations. OPC processes contained a high density of PLs filled with 275 material exhibiting characteristic features of synapses, suggesting that OPCs target specific 276 portions of axons for removal. These ingestions could represent trogocytosis, in which small 277 portions of cells are pinched off ${ }^{50}$. Although the molecular mechanisms that enable trogocytosis 
278 have not been fully defined, OPCs expressed numerous genes involved in phagocytosis. In 279 addition, they contained DNA encoding key neuronal constituents, which due to a higher 280 methylation state are likely to have been acquired from neurons. Together, these data provide 281 evidence that OPCs not only serve as progenitors for oligodendrocytes, but also engage in the 282 pruning of neuronal processes during a robust stage of cortical alteration and maturation. By 283 leveraging multiple glial cell types, the nervous system may accelerate circuit refinement by 284 enhancing overall degradative capacity and enable greater spatial and temporal control of this 285 process by recruiting cells that recognize distinct cellular components.

287 Emerging evidence suggests that OPCs do more than serve as progenitors for oligodendrocytes, as 288 they are found in regions where there is no myelin, and like microglia, OPCs migrate to sites of 289 injury and contribute to scar formation ${ }^{51-53}$. Moreover, recent evidence indicates that OPCs can 290 transform into inflammatory OPCs (iOPCs) that engulf and present exogenous antigens through

291 MHC class I and II when exposed to inflammatory cytokines, suggesting that they may modulate 292 tissue inflammation ${ }^{54,55}$. OPCs share many other features with microglia - they are present at a 293 similar density, maintain a grid-like distribution with non-overlapping domains, possess ramified, 294 radially-oriented processes and are highly motile, continuously exploring their surrounding 295 environment with dynamic filopodia ${ }^{25}$.

The robust dynamics and broad distribution of OPCs place them in an ideal position to engage in 298 the modification of neural circuits that we describe in this study. Remarkably, OPC processes contained a higher density of PLs than surrounding microglia, raising the possibility that they are responsible for a greater amount of pruning at this stage of maturation of the cortex. However, it is also possible the engulfment and digestion of axonal elements proceeds more slowly in OPCs than microglia, leading to more PL accumulation. Although the presence of PLs within OPC processes declined with age, the persistence of OPCs throughout the CNS, which retain a similar 304 morphology and dynamics, raises the possibility that they may retain the capacity to modify circuits and clear cellular debris induced by injury or normal aging.

Many OPCs in this region of the developing cortex will differentiate to form oligodendrocytes that 
309 and OPCs described here are distinct from the early stages of axon wrapping, as these cells had 310 not yet begun transforming into oligodendrocytes, and PL abundance rapidly declined with

311 differentiation. Whether the interactions described here are an early harbinger of myelination ${ }^{56,59}$,

312 to possibly trim superfluous axon branches prior to axon ensheathment or signal which regions of 313 axons are to be myelinated, are not yet known.

315 These findings raise new questions about how distinct glial cell types coordinate their phagocytic 316 behavior to shape brain connectivity and contribute to plasticity, homeostasis and disease

317 processes. Future studies selectively targeting engulfment by OPCs at different time points may 318 reveal the complex role of these progenitors in early refinement of neural circuits and explore their 319 interactions with astrocytes and microglia. 
a
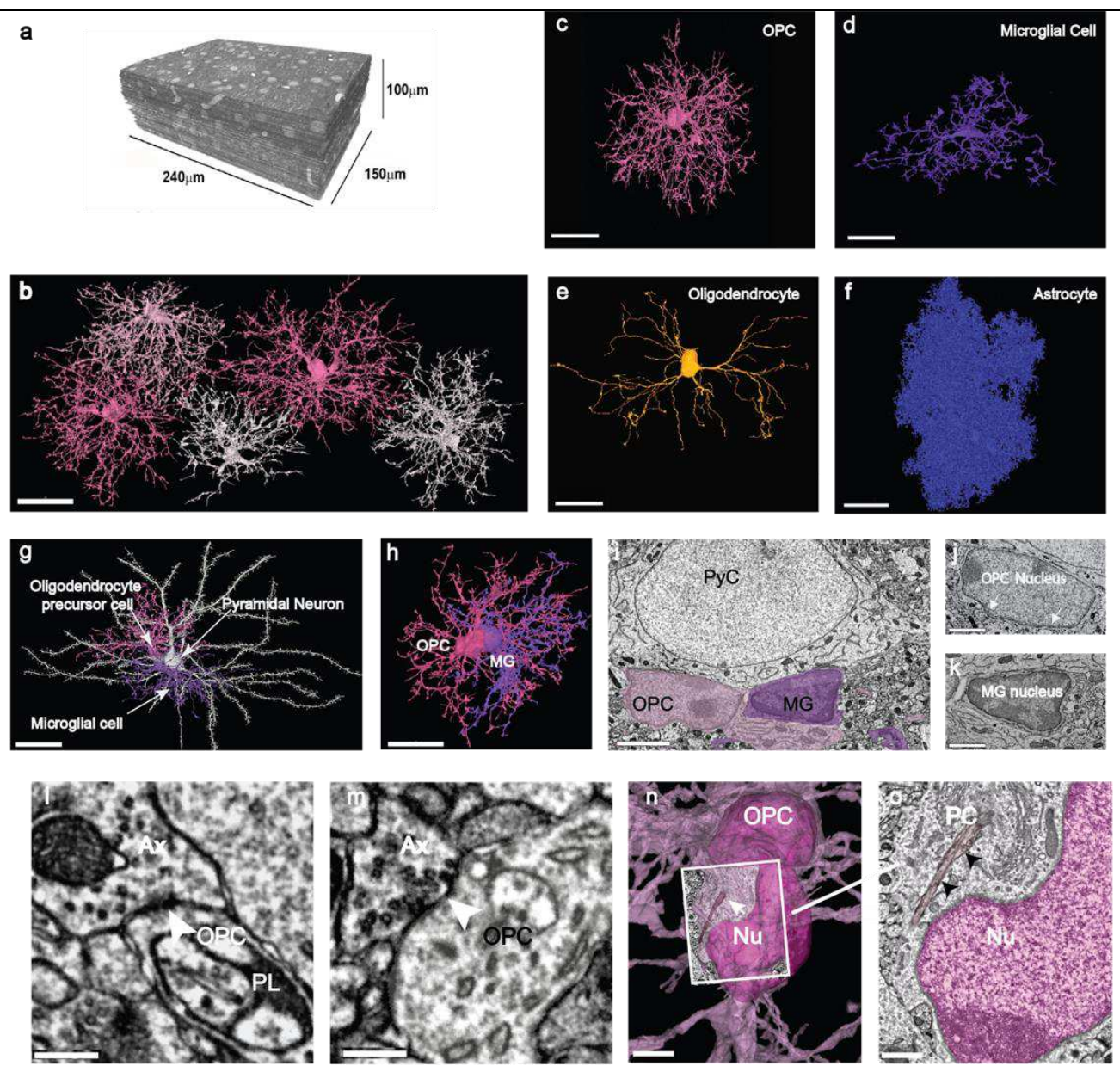

Figure 1: Distinct structural features of OPCs in the developing visual cortex.

a, ssEM reconstruction of $100 \mu \mathrm{m}^{3}$ volume of layer $2 / 3$ mouse visual cortex (P36). b, 3D reconstructions of a subset of OPCs in P36 dataset, showing discrete territories and tiling. Scale bar, $30 \mu \mathrm{m}$. c, 3D rendering of an OPC from P36 dataset showing extensive ramifications emanating from the cell soma. d, 3D rendering of a microglial cell shows its shorter, less branched processes and elongated and flattened soma. e, 3D rendering of a mature myelinating oligodendrocyte in P36 dataset has a smooth and ovoid shaped soma. Note, only the soma and cytoplasmic processes without myelin sheaths are shown. f, 3D rendering of an astrocyte in P36 dataset showing its densely packed cytoplasmic protrusions. Scale bars c-f, $20 \mu \mathrm{m}$. g, 3D rendered pyramidal neuron (white) with an OPC (pink) and a microglial cell (MG, purple) both in satellite positions. Scale bar, $30 \mu \mathrm{m}$. (See also Extended Data Video 1) h, 3D rendering of the same two glial cells in $\mathbf{g}$ shows their close association and intermingling of branches. Scale bar, $20 \mu \mathrm{m}$. i, Ultrathin section slice through an OPC soma (pink) and microglial cell soma (purple). Scale bar, $3 \mu \mathrm{m}$. j, Ultrathin section slice of an OPC nucleus with dense rim of heterochromatin and ruffled edge (arrows). Scale bar, $1.5 \mu \mathrm{m}$. k, Ultrathin section slice of a microglial nucleus showing its dense heterochromatin throughout. Scale bar, $1.5 \mu \mathrm{m}$. Im, Axons (Ax) making synaptic contacts with OPC processes (see also Extended Data Fig.1k). Scale bar, $300 \mathrm{~nm}$. $\mathbf{n}$, The soma of an OPC_(dark pink) bears a primary cilium_(arrow) adjacent to the nucleus (Nu). Scale bar, $3 \mu \mathrm{m}$. o, Ultrathin slice of the boxed area in $\mathbf{n}$ showing the primary cilium (PC)(arrows) close to the OPC nucleus (Nu, dark pink). Scale bar, $750 \mathrm{~nm}$. (See also Extended Data Video 2, for details on how to explore public data). 

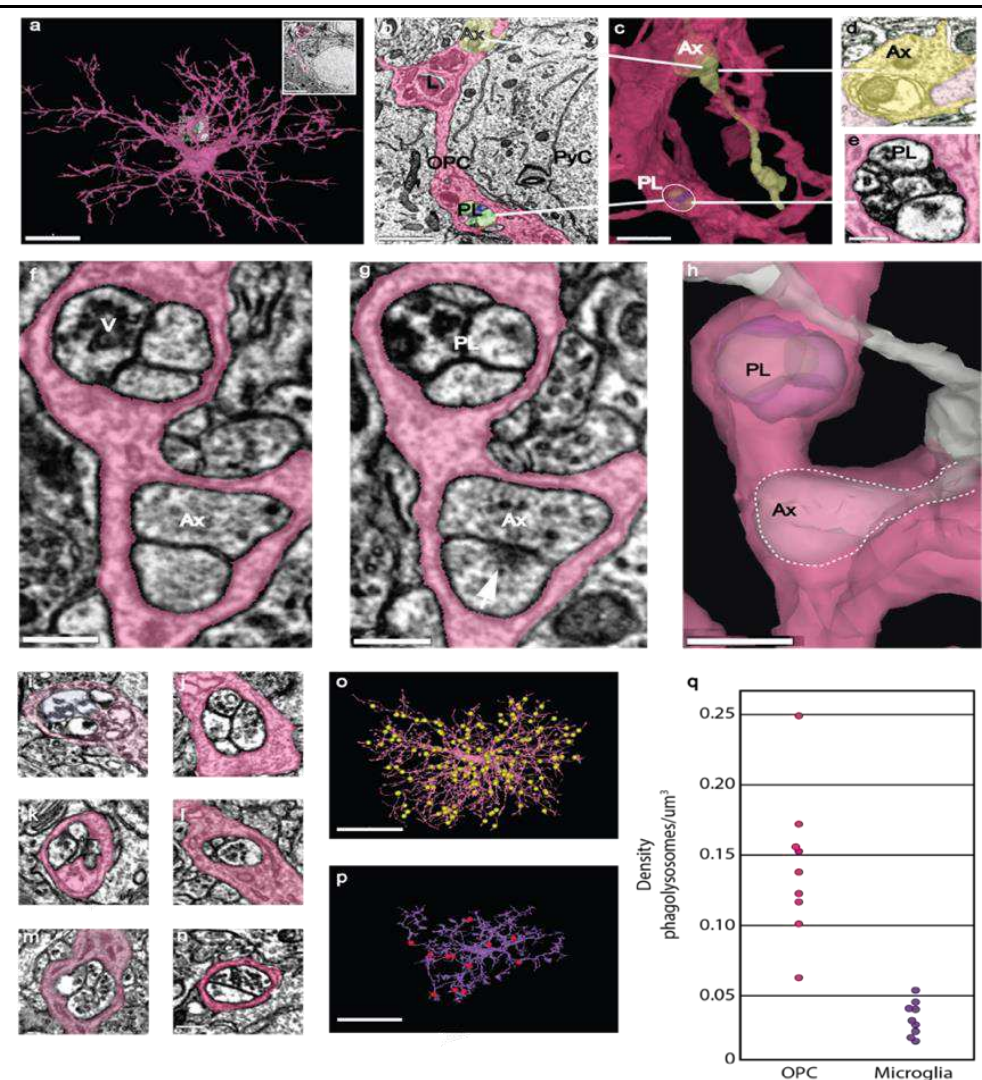

Figure 2: Phagolysosomes are abundant in OPC processes. a, 3D rendering of an OPC from the P36 dataset showing ramified branches. Scale bar, $15 \mu \mathrm{m}$. Insert shows the same area as in b. b. Ultrathin section slice of OPC branch containing a phagolysosome (PL), a lysosome (L) and an axon $(\mathrm{Ax})$ passing through the OPC cytoplasm. The branch contacts the adjacent pyramidal cell (PyC). Scale bar, $1.5 \mu \mathrm{m}$. c, 3D view of the same OPC branch showing the axon (Ax) (yellow) partially encased within the OPC cytoplasm. The same phagolysosome (PL) in $\mathbf{2 b}$ is visible (white circle). Scale bar, $1.5 \mu \mathrm{m}$. d, Higher magnification view of axon in c (yellow). Scale bar, $300 \mathrm{~nm}$. e, Higher magnification of phagolysosome (PL) (white line) in $\mathbf{c}$ highlighting multiple chambers containing cellular debris. Scale bar, 300 $\mathrm{nm}$. f, OPC branch from P36 dataset contains a phagolysosome with vesicles (v) and an encapsulated axon (Ax). Scale bar, $300 \mathrm{~nm}$. g, A portion of the same branch showing the phagolysosome (PL) and post synaptic density of a synapse onto an encapsulated axon (Ax) (arrows). Scale bar, $300 \mathrm{~nm}$. h, 3D rendering of the same branch showing both the phagolysosome (PL) and the axon encapsulated collateral within the OPC cytoplasm. Scale bar, $500 \mathrm{~nm}$. i-n, Examples of PLs from different OPCs showing the presence of $40 \mathrm{~nm}$ vesicles within the chambers. Scale bar, $300 \mathrm{~nm}$. o, 3D reconstruction of an OPC from the P36 dataset. Yellow spheres represent manual annotations of the 189 phagolysosomes found in this OPC. Scale bar, $20 \mu \mathrm{m}$ (original data in http://www.micronsexplorer.org/phagolysosomes/opc). p, 3D reconstruction of a microglial cell from the P36 dataset. Red spheres represent manual annotations of the 13 phagolysosomes present in this cell. Scale bar, $20 \mu \mathrm{m}$ (original data in http://www.micronsexplorer.org/phagolysosomes/microglia). q, Plot comparing PL density in 9 OPCs and 9 microglia in the P36 dataset. 


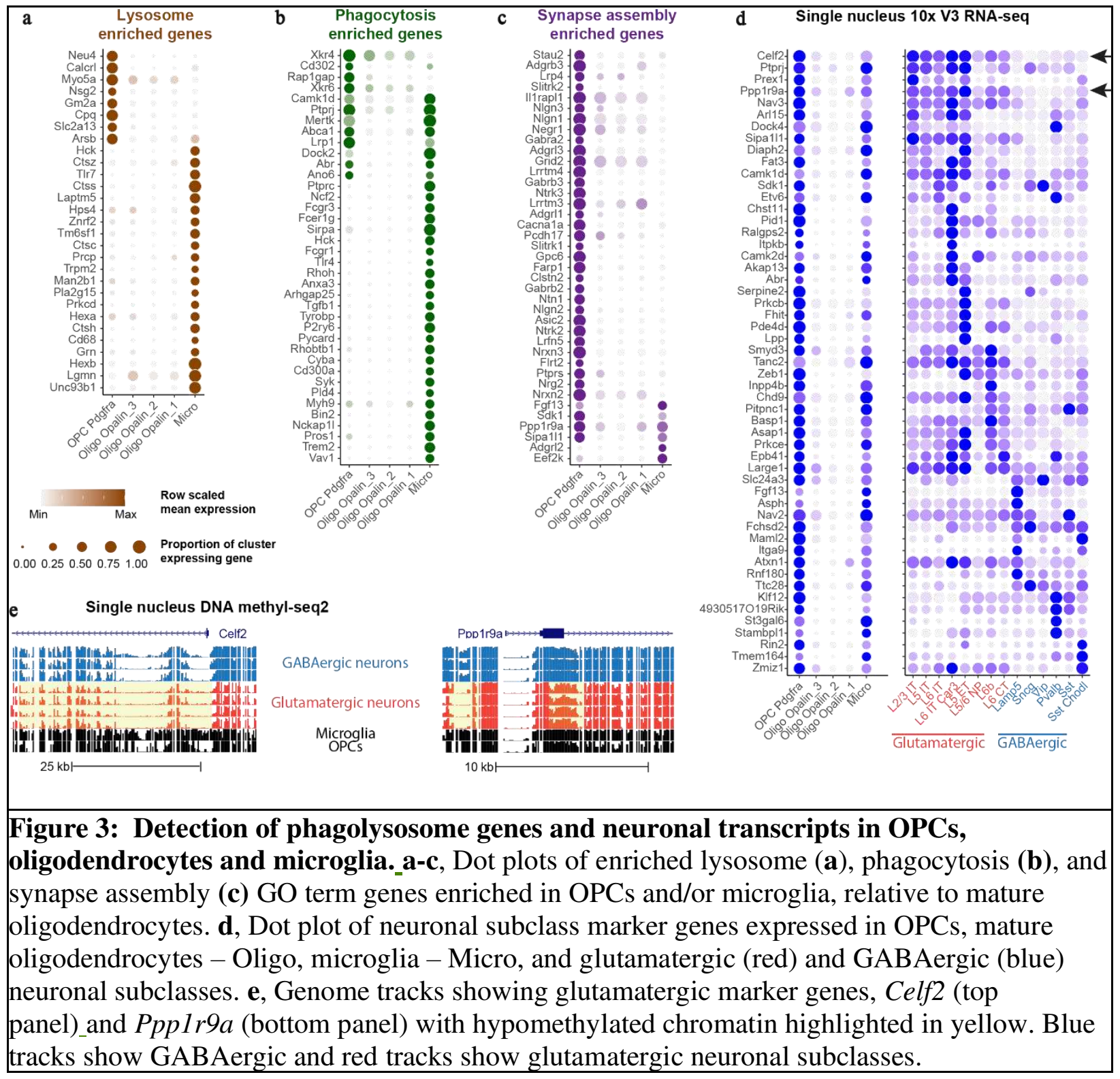



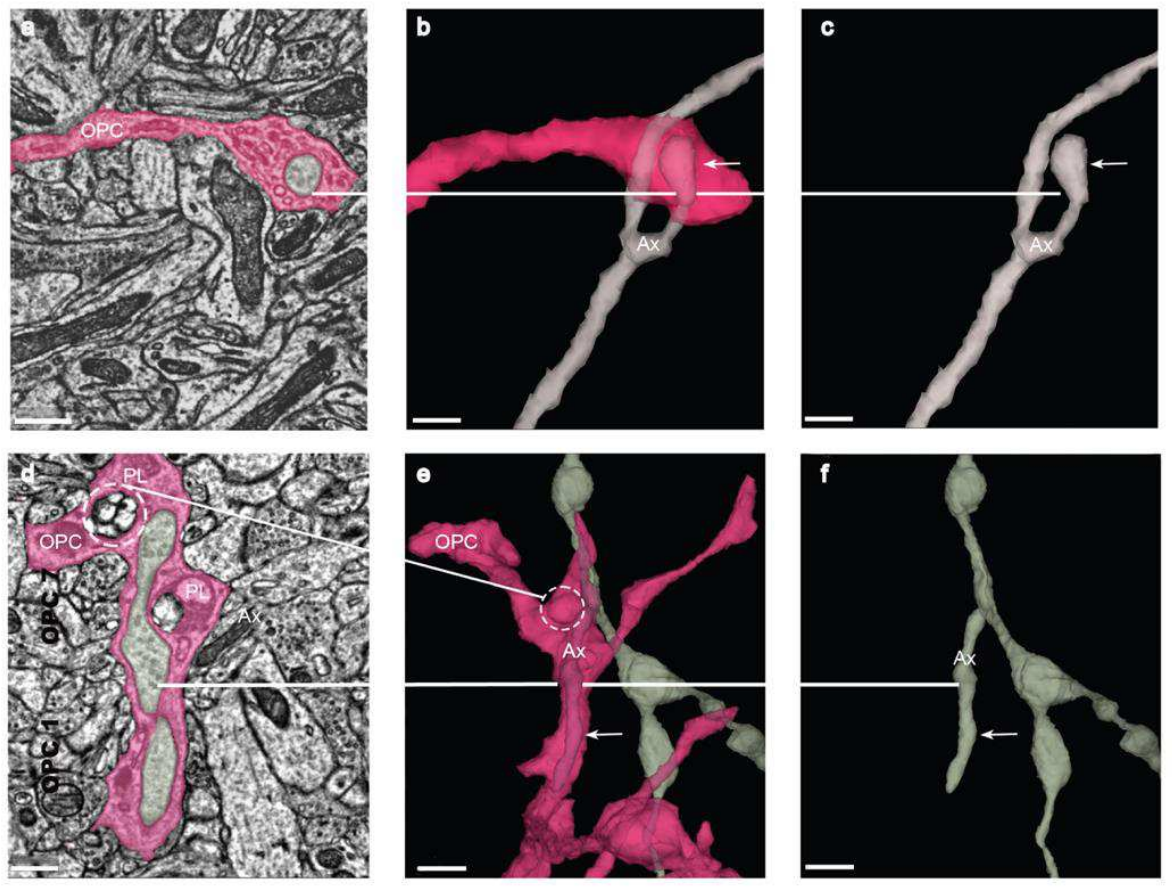

g

h

i
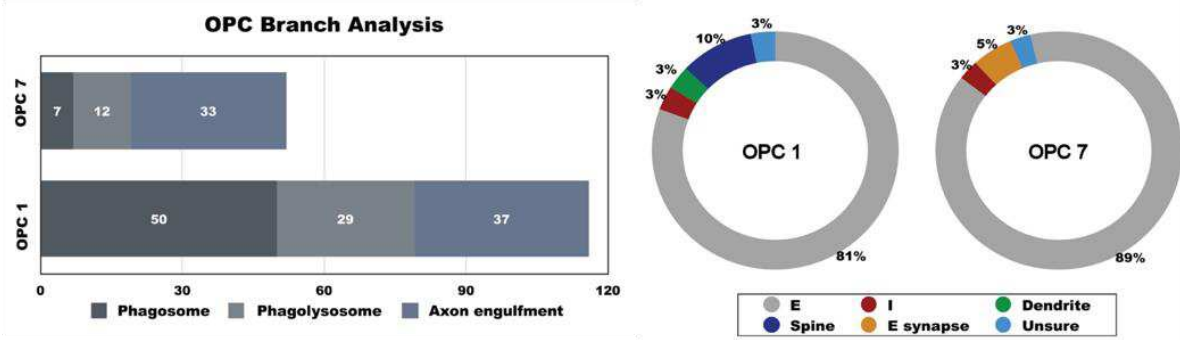

Figure 4: Axon Engulfment by OPCs. a, OPC process in pink ingests an excitatory axon bouton (gray) at its tip. Scale bar, $500 \mathrm{~nm}$. b, 3D reconstruction reveals a small excitatory axon fragment (Ax) encapsulated within the OPC (pink) at the tip (white line, arrow). Scale bar, 500 nm. c, 3D reconstruction shows the same axon (Ax)(arrow) without OPC surrounding it. The bouton tip is visible. Scale bar, $500 \mathrm{~nm}$. d, Ultrathin section slice of a large section of an inhibitory axon (Ax)_collateral branch (white line) in gray ingested within the cytoplasm of the OPC (pink). Two phagolysosomes (PL) (dashed circle) are adjacent to the ingested axon. Scale bar, $500 \mathrm{~nm}$. e, 3D rendered axon collateral branch encapsulated within the cytoplasm of the OPC (arrow, white line) and phagolysosome within the cytoplasm (dashed circle). Scale bar, 1 $\mu \mathrm{m} . \mathbf{f}, 3 \mathrm{D}$ reconstruction of the axon $(\mathrm{Ax})$ in gray shows encapsulated branch (white line, arrow) without the surrounding OPC. Scale bar, $1 \mu \mathrm{m}$. g, Two individual isolated main branches (one each) of OPC cells \#1 and \#7 were analyzed for ingestion events. Ingestions were categorized as phagosome (PS) phagolysosome (PL) or axon collaterals engulfed in OPC cytoplasm. h,i, Donut charts of OPCs 1and 7 quantifies axons partially ingested shows the types-excitatory (E) or inhibitory (I) by examination of boutons and synaptic connections and contact with other neural elements dendrite(D), spine(S), excitatory synapse(Esyn) or unsure (U). Excitatory axons ingestion was most prevalent 89\% in OPC\#1 and 81\% in OPC\#7. (See also Extended Data Video 3) 

Animal procedures were approved by the Institutional Animal Care and Use Committee at the Allen Institute for Brain Science or Baylor College of Medicine.

\section{EM Datasets}

We used two densely segmented and reconstructed volumes, obtained from serial section electron microscopy (ssEM) in mouse primary visual cortex. These data sets were collected as part of the IARPA Machine Intelligence from Cortical Network (MICrONS ) consortium project (https://www.iarpa.gov/index.php/research-programs/microns) to study the functional connectivity of neurons, however they also provide in rich and unprecedented detail the fine morphology of glial cell types. Here we use these datasets to evaluate the population of OPCs and microglia in the 3D environment of the mouse brain visual cortex (preparation methods described below). Dataset 1 was taken from a $200 \mu \mathrm{m}$ thick sample containing layer $2 / 3$ of mouse visual cortex (P36) and measured approximately $250 \mu \mathrm{m}$ x $140 \mu \mathrm{m}$ x $90 \mu \mathrm{m}$ (Fig.1A). Dataset 2 was taken from a $200 \mu \mathrm{m}$ thick sample taken from a P49 mouse visual cortex and included all 6 layers, measuring approximately $56 \mu \mathrm{m} \times 1 \mu \mathrm{mm} \times 30 \mu \mathrm{m}$. The P36 data set is publicly available at https://microns-explorer.org/.

\section{Mouse line}

Mouse P 36 was a triple-heterozygote for the following three genes: (1) Cre driver: CAMKIIa _Cre (Jax: 005359<https://www.jax.org/strain/005359>), (2) tTA driver: B6;CBA- Tg(Camk2atTA)1Mmay/J (Jax: 003010<https://www.jax.org/strain/003010>), (3) GCaMP6f Reporter: Ai93(JAX 024103) (Allen Institute) ${ }^{60}$. Mouse P49 was a cross of: B6;CBA-Tg(Camk2atTA)1Mmay/J (Jax: 003010) and B6;DBA-Tg(tetO-GCaMP6s)2Niell/J (Jax: 024742).

\section{Two-Photon Imaging}

Before preparation for electron microscopy, mice underwent neurophysiology data acquisition conducted at Baylor College of Medicine (details in ${ }^{9}$ ). Note that this 2-photon data was not used in this study. Briefly, a $3 \mathrm{~mm}$ craniotomy was made centered on the primary visual cortex (V1; $2.7 \mathrm{~mm}$ lateral of the midline, contacting the lambda suture), and the cortical window was then sealed with a $3 \mathrm{~mm}$ coverslip (Warner Instruments), using cyanoacrylate glue (VetBond). The mouse was allowed to recover for 1-2 hours prior to the imaging session. Imaging was performed in V1, in a $400 \times 400 \times 200 \mu \mathrm{m} 3$ volume with the superficial surface of the volume at the border of L1 and L2/3, approximately 100um below the pia. Laser excitation was at $920 \mathrm{~nm}$ at $25-45 \mathrm{~mW}$ depending on depth. The objective used was a $25 x$ Nikon objective with a numerical aperture of 1.1 , and the imaging point-spread function was measured with $500 \mathrm{~nm}$ beads and was approximately $0.5 \times 0.5 \times 3 \mu \mathrm{m} 3$ in $\mathrm{x}, \mathrm{y}$, and $\mathrm{z}$. To aid in registration of optical physiology data to EM data, a wide field image of the cranial window visualizing the surface vasculature was provided in addition to a volumetric image stack of the vasculature, encompassing the region of tissue where the neurophysiology dataset was acquired. The vasculature was imaged by subcutaneous injection of $60 \mu \mathrm{L} \mathrm{2.5 \%} \mathrm{Texas} \mathrm{Red} \mathrm{3000MW} \mathrm{Lysine} \mathrm{fixable} \mathrm{(Life} \mathrm{Technologies}$ D3328, allowing blood vessels and GCaMP6-expressing cell bodies to be imaged simultaneously by 2-photon microscopy. Mice were then transferred to the Allen Institute in Seattle and kept in a quarantine facility for 1 to 3 days, prior to perfusion. 


\section{Perfusion}

After induction of anesthesia with isoflurane, the appropriate plane of anesthesia was checked by a lack of toe pinch reflex and the animals were transcardially perfused with $15 \mathrm{ml} 0.15 \mathrm{M}$ cacodylate buffer (EMS, Hatfield, PA, pH 7.4) followed by $30 \mathrm{ml}$ fixative mixture containing 0.08 $\mathrm{M}$ cacodylate ( $\mathrm{pH} 7.4$ ), 2.5\% paraformaldehyde (EMS), $1.25 \%$ glutaraldehyde (EMS) and $2 \mathrm{mM}$ calcium chloride (Sigma). The perfusion solution was based on the work of (Hua et al., 2015). Once the brain was removed it was placed into the same fixative solution to post-fix for 16 to 72 hours at $4{ }^{\circ} \mathrm{C}$.

After perfusion of the animals and excision of the brain, the surface of the cortex was imaged using differential contrast lighting to visualize the surface vasculature of visual cortex and identify the region where the cranial window had previously been. This region was then prepared for Electron Microscopy. Details of the procedures to carefully map the neurophysiology site in the histological sections is described in (details in ${ }^{9}$ ). We omitted these details here as no neurophysiology data was used in this manuscript, even though the anatomical data originates from the same block of tissue that was recorded with two-photon imaging. The brain was washed in $\mathrm{CB}$ (0.1 M cacodylate buffer $\mathrm{pH} 7.4)$ and embedded in $2 \%$ agarose. The agarose was trimmed and mounted for coronal sectioning in a Leica VT1000S vibratome; successive $200 \mu \mathrm{m}$ thick slices were taken until the entire region of cortical tissue previously demarcated by manual markings was sectioned. During this procedure, we also acquired blockface images of each brain slice. The coronal sections containing the imaged site were then selected for histological processing (see below).

\section{EM Histology}

The histology protocol used here is based on the work of ${ }^{61}$ and ${ }^{62}$, with modifications to accommodate different tissue block sizes and to improve tissue contrast for transmission electron microscopy (TEM). Following several washes in CB (0.1 M cacodylate buffer $\mathrm{pH} 7.4)$, the vibratome slices were treated with a heavy metal staining protocol. Initial osmium fixation with $2 \%$ osmium tetroxide in $\mathrm{CB}$ for 90 minutes at room temperature was followed by immersion in $2.5 \%$ potassium ferricyanide in $\mathrm{CB}$ for 90 minutes at room temperature. After $2 \times 30$ minute washes with deionized (DI) water, the tissue was treated with freshly made and filtered $1 \%$ aqueous thiocarbohydrazide at $40{ }^{\circ} \mathrm{C}$ for 10 minutes. The samples were washed $2 \times 30$ minutes with DI water and treated again with $2 \%$ osmium tetroxide in water for 30 minutes at room temperature. Double washes in DI water for 30 min each were followed by immersion in $1 \%$ aqueous uranyl acetate overnight at $4^{\circ}$. The next morning, the samples in the same solution were placed in a heat block to raise the temperature to $50^{\circ}$ for 2 hours. The samples were washed twice in DI water for 30 minutes each, then incubated in Walton's lead aspartate $\mathrm{pH} 5.0$ for 2 hours at $50{ }^{\circ} \mathrm{C}$ in the heat block. After double washes in DI water for 30 minutes each, the slices were dehydrated in an ascending ethanol series $(50 \%, 70 \%, 90 \%, 3 \times 100 \%) 10$ minutes each and two transition fluid steps of $100 \%$ acetonitrile for 20 minutes each. Infiltration with acetonitrile:resin dilutions at 2p:1p (24 h), 1p:1p (48 h) and 1p:2p (24 h) were performed on a gyratory shaker. Samples were placed in $100 \%$ resin for 24 hours, followed by embedment in Hard Plus resin (EMS, Hatfield, PA). The samples were cured in a $60{ }^{\circ} \mathrm{C}$ oven for 96 hours.

In order to evaluate the quality of samples during protocol development and before preparation for large scale sectioning, the following procedure was used for tissue mounting, sectioning and imaging. We evaluated each sample for membrane integrity, overall contrast and quality of ultrastructure. For general tissue evaluation, adjacent slices and tissue sections from the opposite 
hemisphere, processed in the same manner as the ROI slice, were cross-sectioned and thin sections were taken for evaluation of staining throughout the block neighboring the region of interest.

\section{Ultrathin Sectioning}

The tissue block was trimmed to contain the neurophysiology recording site which is the region of interest (ROI) then sectioned to $40 \mathrm{~nm}$ ultrathin sections. For both trimming and sectioning a Leica EM UC7 ultramicrotome was equipped with a diamond trimming tool and an Ultra 35 diamond knife (Diatome USA) respectively. Sectioning speed was set to $0.3 \mathrm{~mm} / \mathrm{sec}$. Eight to ten serial thin sections were cut to form a ribbon, after which the microtome thickness setting was changed to 0 $\mathrm{nm}$ in order to release the ribbon from the knife edge. Then, using an eyelash superglued to a handle, ribbons were organized to pairs and picked up as pairs to copper grids (Pelco, SynapTek, $1.5 \mathrm{~mm}$ slot hole) covered by 50nm thick LUXFilm support (Luxel Corp., Friday Harbor, WA).

\section{Electron microscopy imaging}

The imaging platform used for high throughput serial section imaging is a JEOL-1200EXII 120kV transmission electron microscope that has been modified with an extended column, a custom scintillator, and a large format sCMOS camera outfitted with a low distortion lens. The column extension and scintillator facilitate an estimated 10-fold magnification of the nominal field of view with negligible impact on resolution. Subsequent imaging of the scintillator with a high-resolution, large-format camera allows the capture of fields-of-view as large as $13 \times 13 \mu \mathrm{m}$ at $4 \mathrm{~nm}$ resolution. As with any magnification process, the electron density at the phosphor drops off as the column is extended. To mitigate the impact of reduced electron density on image quality (shot noise), a highsensitivity sCMOS camera was selected and the scintillator composition tuned in order to generate high quality EM images within exposure times of $90-200 \mathrm{~ms}^{63}$.

\section{Image volume assembly and morphological segmentation}

Aligning the individual image tiles and sections into a coherent three-dimensional volume and segmenting the cellular morphology for the P36 dataset was performed as previously described within ${ }^{9-11}$.

\section{Proofreading and Annotation of Volumetric Imagery Data}

We used a combination of Neuroglancer (Maitin-Shepard, https://github.com/google/neuroglancer) and custom tools to annotate and store labeled spatial points ${ }^{64}$. In brief, we used Neuroglancer to simultaneously visualize the imagery and segmentation of the $3 \mathrm{~d}$ EM data. A custom branch of Neuroglancer was developed that could interface with a "dynamic" segmentation database, allowing users to correct errors (i.e., either merging or splitting neurons) in a centralized database from a web browser. Neuroglancer has some annotation functionality, allowing users to place simple annotations during a session, but does not offer a way to store them in a central location for analysis. We thus built a custom cloud-based database system to store arbitrary annotation data centered associated with spatial points that could be propagated dynamically across proofreading events. Annotations were programmatically added to the database using a custom python client and, in relevant cases, after parsing temporary Neuroglancer session states using custom python scripts. These spatial points and their associated data (e.g., synapse type, cell body ID number, or cell types) were linked to stored snapshots of the proofreading for querying and reproducible data analysis. All data analyzed here came from the "v183" snapshot. 


\section{Visualization and Analysis of Mesh Data}

Neuronal meshes were computed by Igneous (https://github.com/seung-lab/igneous) and kept up to date across proofreading. Meshes were analyzed in a custom python library, MeshParty (https://github.com/sdorkenw/MeshParty), that extends Trimesh (https://trimsh.org) with domainspecific features and VTK (https://www.vtk.org) integration for visualization. In cases where skeletons were used, we computed them with a custom modification of the TEASAR algorithm ${ }^{65}$ on the vertex adjacency graph of the mesh object implemented as part of MeshParty. In order to associate annotations such as synapses or AIS boundary points with a mesh, we mapped point annotations to the closest mesh vertex after removing artifacts from the meshing process.

\section{Quantification and Statistical Analysis}

A t-test (two sample assuming unequal variance, two tailed) was performed in Microsoft Excel to compare number and densities of phagolysosomes. Measurements were taken from different cells.

\section{Annotation of cells in the EM volume}

We used a combination of Neuroglancer (Maitin-Shepard https://github.com/google/neuroglancer) and custom tools to annotate and store labeled spatial points. In brief, we used Neuroglancer to simultaneously visualize the imagery and segmentation of the 3d EM data. Neuroglancer incorporates the capability to store and remark on xyz points within the data. These spatial points and their associated data (e.g., Cell type, cell body ID number, or organelles) were linked to stored spreadsheets and documents.

\section{Mouse primary OPC culture}

Cerebral cortices from post-natal day 6 or 7 (P6/P7) CD1 mouse pups (Charles River) were collected, and tissue was dissociated using MACS Milltenyi Biotec Neural Tissue Dissociation Kit (P) (130-092-628), according to the manufacturer's instructions. Briefly, cortices were collected from each pup and incubated at $37^{\circ} \mathrm{C}$ with gentle rotation using a MACSmix ${ }^{\mathrm{TM}}$ Tube Rotator (130090-753) following digestion with pre-warmed enzyme P mix. Cells were then mechanically dissociated and passed through a 70 $\mu \mathrm{m}$ MACS SmartStrainer and a 70 $\mu \mathrm{m}$ Pre-Separation Filter to remove cell clumps before incubation with Anti-O4 Microbeads (130-094-543). Cells were then loaded into an MS column (130-042-201) in for magnetic separation, and positively-selected cells were collected following extrusion through the column.

Cells were plated at a density of about 20,000 cells / well in a 24-well plate with $1.5 \mathrm{~mm}$ coverslips in each well that had previously been coated overnight with poly-d-lysine $(50 \mu \mathrm{L}$ PDL in $250 \mathrm{~mL}$ $\mathrm{dH}_{2} \mathrm{O}$ ). Cells were expanded overnight at $37^{\circ} \mathrm{C}$ in OPC media containing recombinant human PDGF-AA and Neurotrophin-3 until optimal density was reached. The plates were then processed for immunofluorescence staining.

\section{Staining of primary OPC cultures}

Coverslips were washed once with 1x PBS before 4\% PFA was applied for 13 minutes at room temperature. Cells were then washed twice with 1x PBS (5 minutes/wash) before blocking in $0.5 \%$ Saponin, 5\% normal donkey serum, and 1x PBS) for 1 hour at room temperature. Cells were then stained with primary antibodies against NG2 proteoglycan (guinea pig, 1:100, custom Bergles 
antibody), LAMP2 (rat, Abcam, 1:200) to visualize OPC cell bodies/ processes and lysosomes, respectively. Following overnight incubation at $4{ }^{\circ} \mathrm{C}$ with rotation, cells were washed three times with 1x PBS (5 minutes/wash) at room temperature. AlexaFluor secondary antibodies (488 donkey anti-rat and 647 donkey anti-guinea pig) were applied for 1 hour at room temperature in the dark. Cells were again washed three times, DAPI stain was applied for 10 minutes, and cells were washed again twice before coverslips were removed and mounted to slides using ProLong ${ }^{\mathrm{TM}}$ Gold

\section{Imaging and analysis of primary OPC cultures}

Cells from three separate culture experiments were used for the analysis. All images were taken at $63 x$ magnification using the AiryScan function on a Zeiss 710 confocal microscope. Airyscanprocessed z-stacks of $\mathrm{NG}^{+}$individual OPCs were projected using the maximum intensity projection function, and the number of LAMP2 ${ }^{+}$vesicles within cell processes were quantified.

\section{Reagents used in primary OPC cultures}

OPC Culture Tools - all Miltenyi (Cat no.)

MACS Milltenyi Biotec Neural Tissue Dissociation Kit (P) (130-092-628)

MACS Milltenyi Biotec Anti-O4 MicroBeads (130-094-543)

MACS SmartStrainer $(70 \mu \mathrm{m})(130-090-753)$

MACSmix ${ }^{\mathrm{TM}}$ Tube Rotator (130-090-753)

Pre-Separation Filters, 70 $\mu \mathrm{m}$ (130-095-823)

MACS MS Columns (130-042-201)

MACS Multistand (130-042-303)

MiniMACS ${ }^{\mathrm{TM}}$ Separator (130-042-102)

\section{Antibodies}

Anti-NG2 (Guinea pig, source: Bergles Laboratory)

Anti-LAMP2 (Abcam, cat no. ab13524)

\section{Additional Reagent}

0.5\% saponin (Millipore Sigma, 47036)

ProLong ${ }^{\mathrm{TM}}$ Gold Antifade Mountant (ThermoFisher, cat no. P10144)

\section{Experimental Models: Organisms/ Strains}

CD1 mice, Charles River (Strain number 022)

\section{Molecular analysis of OPCs and microglia with single nucleus RNA-seq and single nucleus DNA methyl-seq2 dataset.}

We analyzed recently described Chromium 10x V3 single nucleus RNA-seq and single nucleus DNA methyl-seq2 datasets from mouse primary motor cortex ${ }^{35}$, available from the Neuroscience Multi-omic Data Archive (NeMO, https://assets.nemoarchive.org/dat-ch1nqb7). Only the 159,738 nuclei from the dataset generated by the Broad Institute were used.

For gene expression dot plots, the mean UMIs per cluster and proportion of cluster with UMIs greater than 1 were calculated using the scrattch.hicat v0.0.22 library (https://github.com/AllenInstitute/scrattch.hicat) and visualized using ggplots2 v3.3.3 library ${ }^{66}$ in $\mathrm{R}$ v3.4.1. Mean expression was scaled from 0 to 1 for each gene for each major cell class (i.e. 
non-neuronal and neurons). To find neuronal subclass marker genes, we first created a Seurat ${ }^{67}$, $565{ }^{68}$ object of only the neuronal cell types, downsampled to 500 nuclei per neuronal subclass. We

566 then used the FindAllMarkers function from Seurat v3.2.0 with the 'roc' test to identify

567 differentially expressed genes that were enriched in a particular neuronal subclass compared to

568 other neurons. Neuronal subclass marker genes were then filtered to include genes with greater 569 than $2 \log 2 \mathrm{FC}$ in OPCs and microglia relative to each mature oligodendrocyte cluster (Oligo

570 Opalin_1-3), and with greater than $2 \log 2$ cpm expression in OPCs and microglia.

571 To visualize single nucleus DNA methyl-seq2 tracks, we loaded gene body methylation (CGN)

572 tracks into the UCSC genome browser. Example tracks of neuronal subclass marker genes that

573 showed expression in OPCs were identified to highlight the lack of DNA hypomethylation in

574 OPCs.

575 Specific GO term gene lists for lysosome, phagocytosis, and synapse assembly were downloaded from http://www.informatics.jax.org/go/term/ on January $20^{\text {th }}, 2021^{69,70,71}$. Each gene list was filtered to include genes with greater than $2 \log 2 \mathrm{FC}$ in OPCs and/or microglia relative to each mature oligodendrocyte cluster, and with greater than $2 \log 2 \mathrm{cpm}$ expression in OPCs and/or microglia.

\section{Data availability}

The raw images, segmentation, and synaptic connectivity will be made available upon or before publication.

\section{Code availability}

All software is open source and available at http://github.com/seung-lab if not otherwise mentioned.

Alembic: Stitching and alignment.

CloudVolume: Reading and writing volumetric data, meshes, and skeletons to and from the cloud

Chunkflow: Running convolutional nets on large datasets

DeepEM: Training convolutional nets to detect neuronal boundaries.

DynamicAnnotationFramework: Proofreading and connectome updates

(visit https://github.com/seung-lab/AnnotationPipelineOverview for repository list)

Igneous: Coordinating downsampling, meshing, and data management.

MeshParty: Interaction with meshes and mesh-based skeletonization

(https://github.com/sdorkenw/MeshParty)

MMAAPP: Watershed, size-dependent single linkage clustering, and mean affinity agglomeration.

605

606

607

608

609 
610 
6131 Pan, Y. \& Monje, M. Activity Shapes Neural Circuit Form and Function: A Historical

614 Perspective. J Neurosci 40, 944-954, doi:10.1523/JNEUROSCI.0740-19.2019 (2020).

6152 Schafer, D. P. et al. Microglia sculpt postnatal neural circuits in an activity and 616 complement-dependent manner. Neuron 74, 691-705, doi:10.1016/j.neuron.2012.03.026 (2012).

6173 Chung, W. S. et al. Astrocytes mediate synapse elimination through MEGF10 and 618 MERTK pathways. Nature 504, 394-400, doi:10.1038/nature12776 (2013).

6194 Raff, M. C., Miller, R. H. \& Noble, M. A glial progenitor cell that develops in vitro into 620 an astrocyte or an oligodendrocyte depending on culture medium. Nature 303, 390-396,

621 doi:10.1038/303390a0 (1983).

6225 Baxi, E. G. et al. Lineage tracing reveals dynamic changes in oligodendrocyte precursor 623 cells following cuprizone-induced demyelination. Glia 65, 2087-2098, doi:10.1002/glia.23229 624 (2017).

6256 Bergles, D. \& Jahr, C. E. Glutamatergic synapses on oligodendrocyte precursor cells in 626 the hippocampus. Nature 405, doi:0.1016/s0896-6273(00)80420-1

627 (1997).

6287 Lin, S. C. \& Bergles, D. E. Synaptic signaling between neurons and glia. Glia 47, 290629 298, doi:10.1002/glia.20060 (2004).

6308 Bergles, D. E. \& Richardson, W. D. Oligodendrocyte Development and Plasticity. Cold 631 Spring Harb Perspect Biol 8, a020453, doi:10.1101/cshperspect.a020453 (2015).

6329 Dorkenwald, S. et al. Binary and analog variation of synapses between cortical pyramidal 633 neurons

634 . bioRxiv, doi:10.1101/2019.12.29.890319 (2019).

63510 Schneider-Mizell, C. M. et al. Chandelier cell anatomy and function reveal a variably 636 distributed but common signal. bioRxiv, doi:10.1101/2020.03.31.018952 (2020).

63711 Turner, N. L. et al. Multiscale and multimodal reconstruction of cortical structure and 638 function. bioRxiv, doi:10.1101/2020.10.14.338681 (2020).

63912 Motta, A. et al. Dense connectomic reconstruction in layer 4 of the somatosensory cortex. 640 Science 366, doi:10.1126/science.aay3134 (2019).

64113 Mori, S. \& Leblond, C. P. Electron microscopic identification of three classes of 642 oligodendrocytes and a preliminary study of their proliferative activity in the corpus callosum of 643 young rats. J Comp Neurol 139, 1-28, doi:10.1002/cne.901390102 (1970).

64414 Dawson, M. R. L., J.M.; Reynolds, R. NG2-expressing cells in the CNS. J. Neurosci res 645 61, 471-479, doi:DOI: 10.1002/1097-4547(20000901)61:5<471::AID-JNR1>3.0.CO;2-N 
64715 Ong, W. Y. \& Levine, J. M. A light and electron microscopic study of NG2 chondroitin 648 sulfate proteoglycan-positive oligodendrocyte precursor cells in the normal and kainate-lesioned 649 rat hippocampus. Neuroscience 92, 83-95, doi:10.1016/s0306-4522(98)00751-9 (1999).

65016 Peters, A. A fourth type of neurogial cell in the adult central nervous system. Journal of 651 Neurocytology 33, 345-357, doi:https://doi.org/10.1023/B:NEUR.0000044195.64009.27

652 (2004).

65317 Vaughn, J. E., Hinds, P. L. \& Skoff, R. P. Electron microscopic studies of Wallerian 654 degeneration in rat optic nerves. I. The multipotential glia. J Comp Neurol 140, 175-206, 655 doi:10.1002/cne.901400204 (1970).

$65618 \quad$ Butt, A. M. et al. Cells expressing the NG2 antigen contact nodes of Ranvier in adult 657 CNS white matter. Glia 26, 84-91 (1999).

65819 Bushong, E. A., Martone, M. E., Jones, Y. Z. \& Ellisman, M. H. Protoplasmic astrocytes 659 in CA1 stratum radiatum occupy separate anatomical domains. Vol. 22 (2002).

66020 Nimmerjahn, A., Kirchhoff, F. \& Helmchen, F. Resting microglial cells are highly 661 dynamic surveillants of brain parenchyma in vivo. Science 308, 1314-1318, 662 doi:10.1126/science.1110647 (2005).

$66321 \quad$ Ventura, R. \& Harris, K. M. Three-Dimensional Relationships between Hippocampal 664 Synapses and Astrocytes. The Journal of Neuroscience 19, 6897-6906, doi:10.1523/jneurosci.19665 16-06897.1999 (1999).

66622 Schafer, D. P., Lehrman, E. K. \& Stevens, B. The "quad-partite" synapse: microglia667 synapse interactions in the developing and mature CNS. Glia 61, 24-36, doi:10.1002/glia.22389 668 (2013).

66923 Battefeld, A., Klooster, J. \& Kole, M. H. Myelinating satellite oligodendrocytes are 670 integrated in a glial syncytium constraining neuronal high-frequency activity. Nat Commun 7 , 671 11298, doi:10.1038/ncomms11298 (2016).

67224 Wogram, E. et al. Satellite microglia show spontaneous electrical activity that is 673 uncorrelated with activity of the attached neuron. Eur J Neurosci 43, 1523-1534, 674 doi:10.1111/ejn.13256 (2016).

67525 Hughes, E. G., Kang, S. H., Fukaya, M. \& Bergles, D. E. Oligodendrocyte progenitors 676 balance growth with self-repulsion to achieve homeostasis in the adult brain. Nat Neurosci 16, 677 668-676, doi:10.1038/nn.3390 (2013).

$67826 \quad$ Kinchen, J. M. \& Ravichandran, K. S. Phagosome maturation: going through the acid 679 test. Nat Rev Mol Cell Biol 9, 781-795, doi:10.1038/nrm2515 (2008). 

maturation, and resolution. Immunol Rev 273, 156-179, doi:10.1111/imr.12439 (2016).

68228 Tremblay, M. E., Lowery, R. L. \& Majewska, A. K. Microglial interactions with 683 synapses are modulated by visual experience. PLoS Biol 8, e1000527, 684 doi:10.1371/journal.pbio.1000527 (2010).

68529 Huynh, K. K. et al. LAMP proteins are required for fusion of lysosomes with 686 phagosomes. EMBO J 26, 313-324, doi:10.1038/sj.emboj.7601511 (2007).

$68730 \quad$ Sipe, G. O. et al. Microglial P2Y12 is necessary for synaptic plasticity in mouse visual 688 cortex. Nat Commun 7, 10905, doi:10.1038/ncomms10905 (2016).

68931 Paolicelli, R. C. et al. Synaptic pruning by microglia is necessary for normal brain 690 development. Science 333, 1456-1458, doi:10.1126/science.1202529 (2011).

69132 Peri, F. \& Nusslein-Volhard, C. Live imaging of neuronal degradation by microglia 692 reveals a role for v0-ATPase a1 in phagosomal fusion in vivo. Cell 133, 916-927, 693 doi:10.1016/j.cell.2008.04.037 (2008).

69433 Espinosa, J. S. \& Stryker, M. P. Development and plasticity of the primary visual cortex. 695 Neuron 75, 230-249, doi:10.1016/j.neuron.2012.06.009 (2012).

$69634 \quad$ Yoshimura, K. \& Takeda, S. Hedgehog signaling regulates myelination in the peripheral 697 nervous system through primary cilia. Differentiation 83, S78-85, doi:10.1016/j.diff.2011.10.006 698 (2012).

69935 Yao, Z. et al. A taxonomy of transcriptomic cell types across the isocortex and 700 hippocampal formation. Cell, 2020.2003.2030.015214, doi:10.1016/j.cell.2021.04.021 (2021).

70136 Mo, A. et al. Epigenomic Signatures of Neuronal Diversity in the Mammalian Brain. 702 Neuron 86, 1369-1384, doi:10.1016/j.neuron.2015.05.018 (2015).

70337 Schneble, N. et al. The protein-tyrosine phosphatase DEP-1 promotes migration and 704 phagocytic activity of microglial cells in part through negative regulation of fyn tyrosine kinase.

705 Glia 65, 416-428, doi:10.1002/glia.23100 (2017).

70638 Fernandez-Castaneda, A. et al. The active contribution of OPCs to neuroinflammation is 707 mediated by LRP1. Acta Neuropathol 139, 365-382, doi:10.1007/s00401-019-02073-1 (2020).

70839 Fitzer-Attas, C. J. et al. Fcgamma receptor-mediated phagocytosis in macrophages 709 lacking the Src family tyrosine kinases Hck, Fgr, and Lyn. J Exp Med 191, 669-682, 710 doi:10.1084/jem.191.4.669 (2000).

$71140 \quad$ Suzuki, J., Imanishi, E. \& Nagata, S. Exposure of phosphatidylserine by Xk-related 712 protein family members during apoptosis. J Biol Chem 289, 30257-30267,

713 doi:10.1074/jbc.M114.583419 (2014). 
$71441 \quad$ Simons, M. \& Nave, K. A. Oligodendrocytes: Myelination and Axonal Support. Cold

715 Spring Harb Perspect Biol 8, a020479, doi:10.1101/cshperspect.a020479 (2015).

71642 Snaidero, N. \& Simons, M. Myelination at a glance. J Cell Sci 127, 2999-3004,

717 doi:10.1242/jcs.151043 (2014).

71843 Micheva, K. D. et al. A large fraction of neocortical myelin ensheathes axons of local

719 inhibitory neurons. Elife 5, doi:10.7554/eLife.15784 (2016).

$72044 \quad$ Stogsdill, J. A. \& Eroglu, C. The interplay between neurons and glia in synapse 721 development and plasticity. Curr Opin Neurobiol 42, 1-8, doi:10.1016/j.conb.2016.09.016

722 (2017).

$72345 \mathrm{Wu}$, X. et al. GABA signaling promotes synapse elimination and axon pruning in 724 developing cortical inhibitory interneurons. J Neurosci 32, 331-343,

725 doi:10.1523/JNEUROSCI.3189-11.2012 (2012).

72646 Raiders, S. et al. Engulfed by Glia: Glial Pruning in Development, Function, and Injury 727 across Species. J Neurosci, doi:10.1523/JNEUROSCI.1660-20.2020 (2021).

72847 Bishop, D. L., Misgeld, T., Walsh, M. K., Gan, W. B. \& Lichtman, J. W. Axon branch 729 removal at developing synapses by axosome shedding. Neuron 44, 651-661,

730 doi:10.1016/j.neuron.2004.10.026 (2004).

73148 Sierra, A., Abiega, O., Shahraz, A. \& Neumann, H. Janus-faced microglia: beneficial and 732 detrimental consequences of microglial phagocytosis. Front Cell Neurosci 7, 6,

733 doi:10.3389/fncel.2013.00006 (2013).

73449 Lee, J. H. et al. Astrocytes phagocytose adult hippocampal synapses for circuit

735 homeostasis. Nature, doi:10.1038/s41586-020-03060-3 (2020).

$73650 \quad$ Lim, T. K. \& Ruthazer, E. S. Microglial trogocytosis and the complement system 737 regulate axonal pruning in vivo. Elife 10, doi:10.7554/eLife.62167 (2021).

73851 Tan, A. M., Zhang, W. \& Levine, J. M. NG2: a component of the glial scar that inhibits 739 axon growth. J Anat 207, 717-725, doi:10.1111/j.1469-7580.2005.00452.x (2005).

$74052 \quad$ Hesp, Z. C. et al. Proliferating NG2-Cell-Dependent Angiogenesis and Scar Formation 741 Alter Axon Growth and Functional Recovery After Spinal Cord Injury in Mice. J Neurosci 38, 742 1366-1382, doi:10.1523/JNEUROSCI.3953-16.2017 (2018).

74353 Jin, X., Riew, T. R., Kim, H. L., Choi, J. H. \& Lee, M. Y. Morphological characterization 744 of NG2 glia and their association with neuroglial cells in the 3-nitropropionic acid-lesioned 745 striatum of rat. Sci Rep 8, 5942, doi:10.1038/s41598-018-24385-0 (2018).

74654 Kirby, L. et al. Oligodendrocyte precursor cells present antigen and are cytotoxic targets 747 in inflammatory demyelination. Nat Commun 10, 3887, doi:10.1038/s41467-019-11638-3

748 (2019). 
55 Falcao, A. M. et al. Disease-specific oligodendrocyte lineage cells arise in multiple 750 sclerosis. Nat Med 24, 1837-1844, doi:10.1038/s41591-018-0236-y (2018).

$75156 \quad$ Barres, B. A. \& Raff, M. C. Axonal control of oligodendrocyte development. J Cell Biol 752 147, 1123-1128, doi:10.1083/jcb.147.6.1123 (1999).

75357 Tomassy, G. S. et al. Distinct profiles of myelin distribution along single axons of 754 pyramidal neurons in the neocortex. Science 344, 319-324, doi:10.1126/science.1249766 (2014).

75558 Timmler, S. \& Simons, M. Grey matter myelination. Glia 67, 2063-2070, 756 doi:10.1002/glia.23614 (2019).

75759 Wake, H. et al. Nonsynaptic junctions on myelinating glia promote preferential 758 myelination of electrically active axons. Nat Commun 6, 7844, doi:10.1038/ncomms8844 (2015).

75960 Madisen, L. et al. Transgenic mice for intersectional targeting of neural sensors and 760 effectors with high specificity and performance. Neuron $\mathbf{8 5}, 942-958$, 761 doi:10.1016/j.neuron.2015.02.022 (2015).

76261 Hua, Y., Laserstein, P. \& Helmstaedter, M. Large-volume en-bloc staining for electron 763 microscopy-based connectomics. Nat Commun 6, 7923, doi:10.1038/ncomms8923 (2015).

76462 Tapia, J. C. et al. High-contrast en bloc staining of neuronal tissue for field emission 765 scanning electron microscopy. Nat Protoc 7, 193-206, doi:10.1038/nprot.2011.439 (2012).

76663 Yin, W. et al. A petascale automated imaging pipeline for mapping neuronal circuits with 767 high-throughput transmission electron microscopy. Nat Commun 11, 4949, doi:10.1038/s41467768 020-18659-3 (2020).

76964 Wu, J., Silversmith, W. M., Lee, K. \& Seung, H. S. Chunkflow: hybrid cloud processing 770 of large 3D images by convolutional nets. Nature Methods 18, 328-330, doi:10.1038/s41592771 021-01088-5 (2021).

77265 M. Sato, I. B., M. A. Bender, A. E. Kaufman and M. Nakajima. TEASAR- Tree-structure 773 Extraction Algorithm for Accurate and Robust Skeletons Proceedings the Eighth Pacific

774 Conference on Computer Graphics and Applications, 281-449,

775 doi:10.1109/PCCGA.2000.883951 (2000).

77666 Wickham, H. \& Grolemund, G. R for Data Science: Import, Tidy, Transform, Visualize, 777 and Model Data. (O'Reilly Media, 2016).

77867 Stuart, T. et al. Comprehensive Integration of Single-Cell Data. Cell 177, 1888-1902 779 e1821, doi:10.1016/j.cell.2019.05.031 (2019).

78068 Butler, A., Hoffman, P., Smibert, P., Papalexi, E. \& Satija, R. Integrating single-cell 781 transcriptomic data across different conditions, technologies, and species. Nat Biotechnol 36, 782 411-420, doi:10.1038/nbt.4096 (2018). 
$78369 \quad$ Bult, C. J. et al. Mouse Genome Database (MGD) 2019. Nucleic Acids Res 47, D801784 D806, doi:10.1093/nar/gky1056 (2019).

$78570 \quad$ Smith, C. M. et al. The mouse Gene Expression Database (GXD): 2019 update. Nucleic 786 Acids Res 47, D774-D779, doi:10.1093/nar/gky922 (2019).

$78771 \quad$ Krupke, D. M. et al. The Mouse Tumor Biology Database: A Comprehensive Resource 788 for Mouse Models of Human Cancer. Cancer Res 77, e67-e70, doi:10.1158/0008-5472.CAN-177890584 (2017).

790

791

792

793

794

795

796 


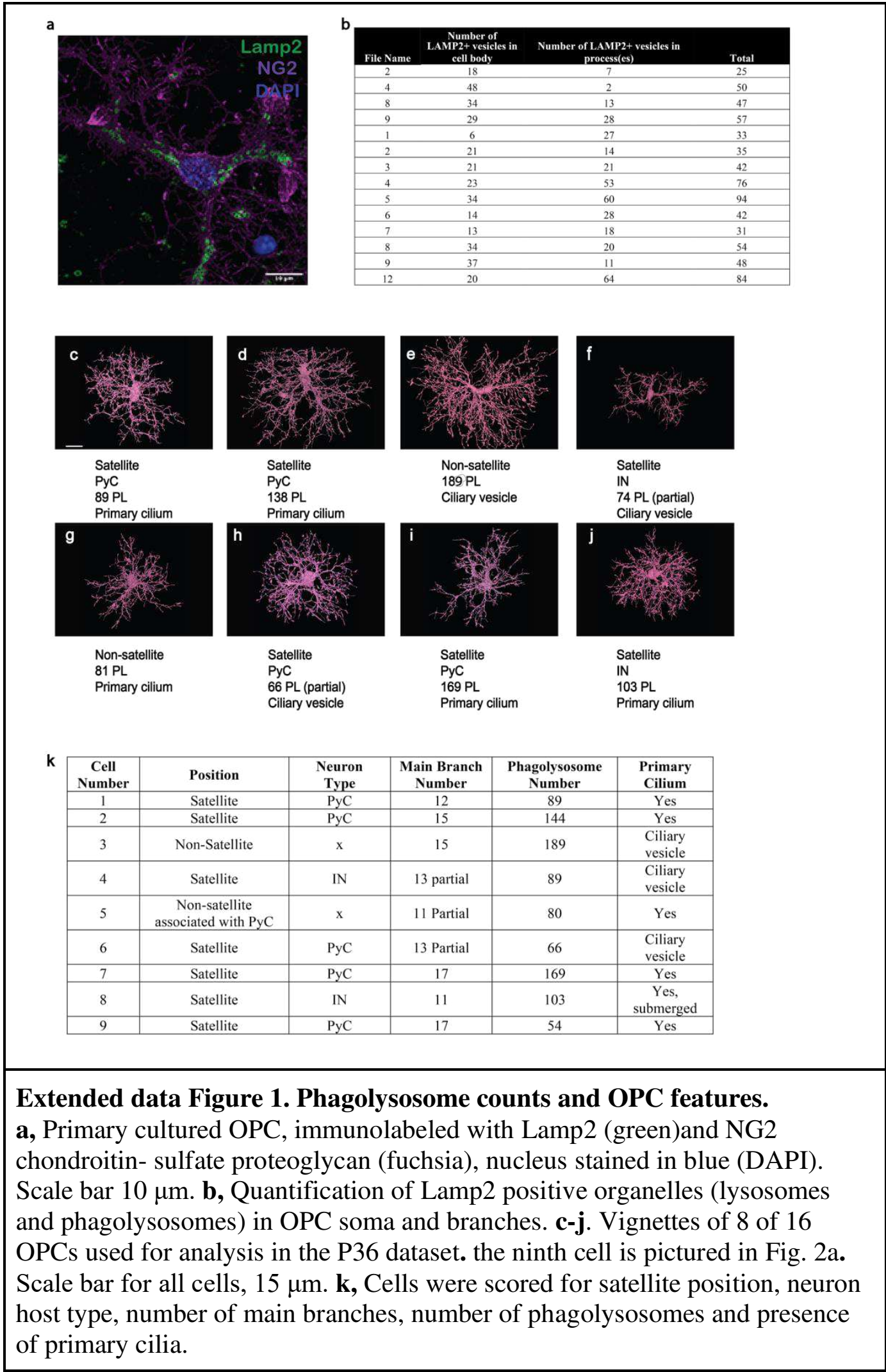




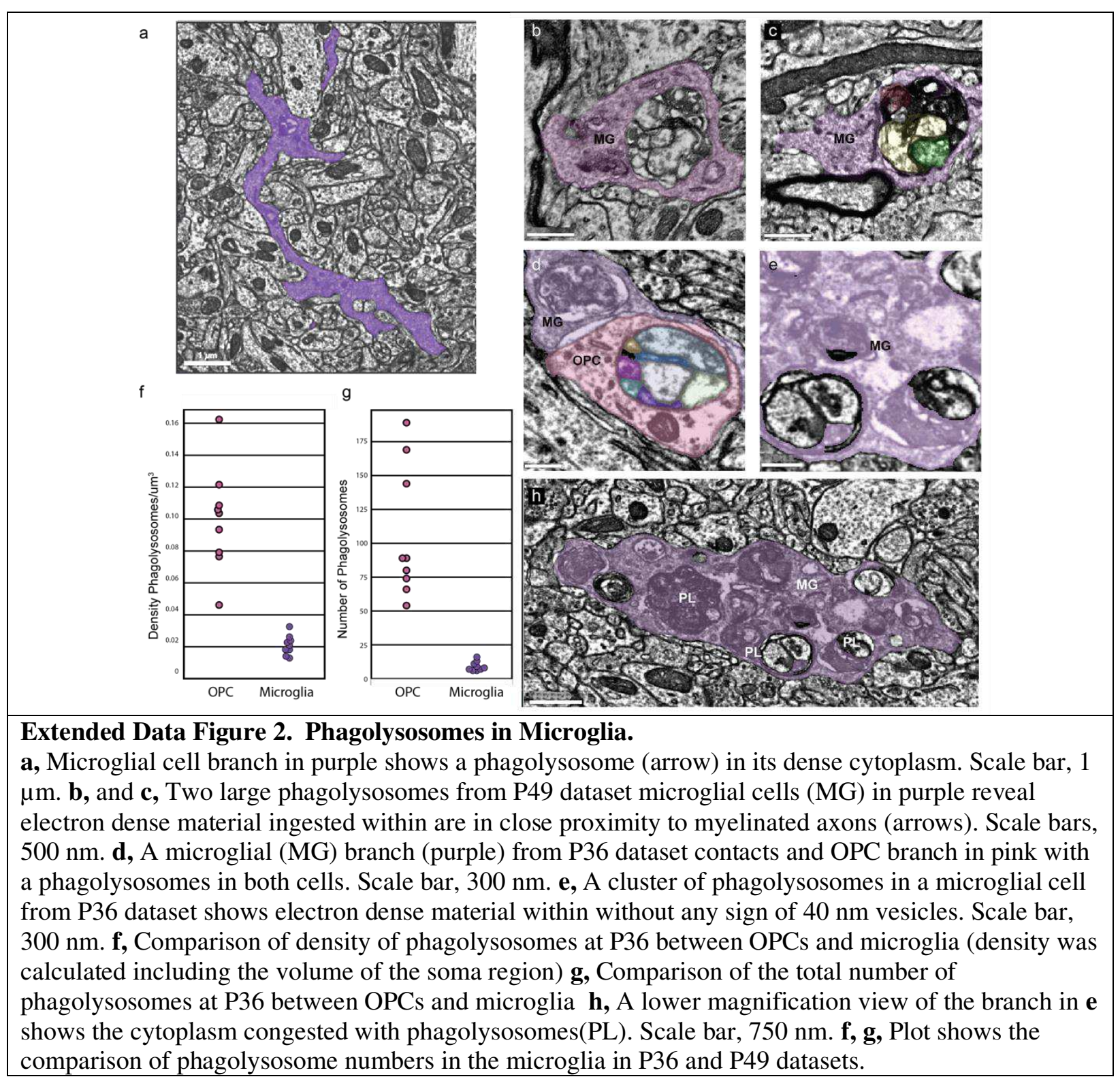



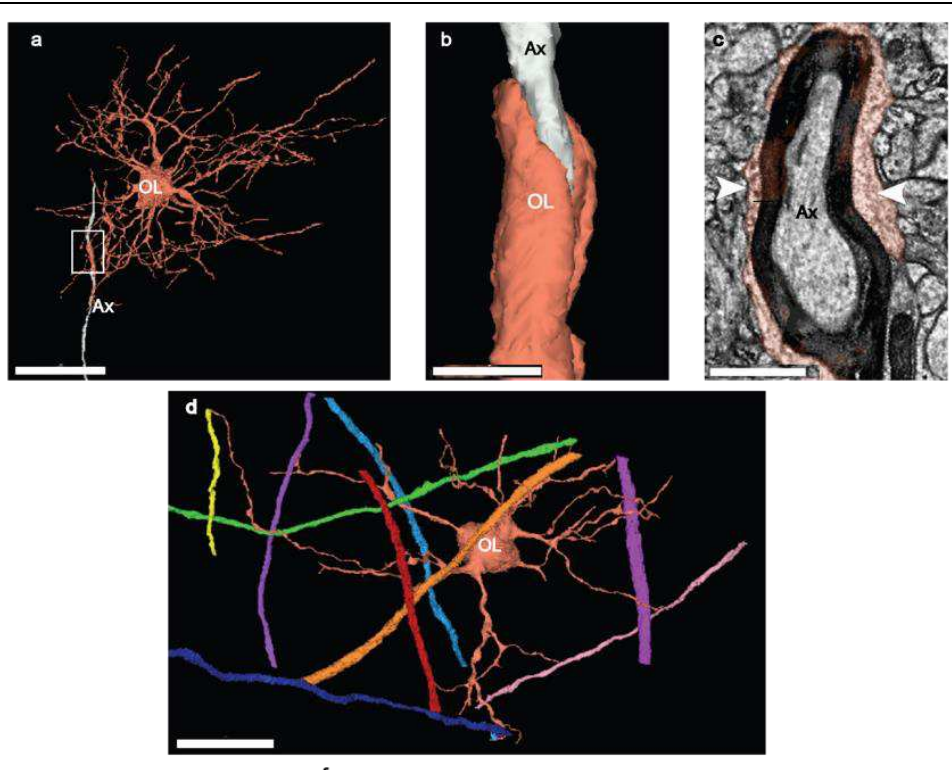

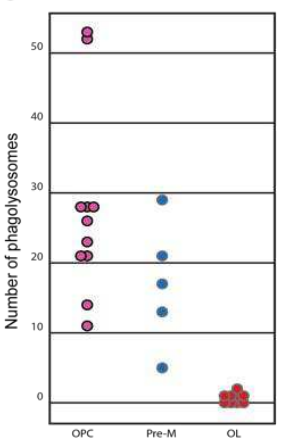

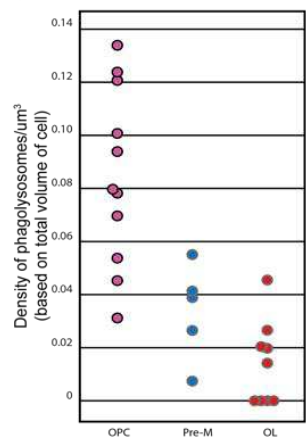

g

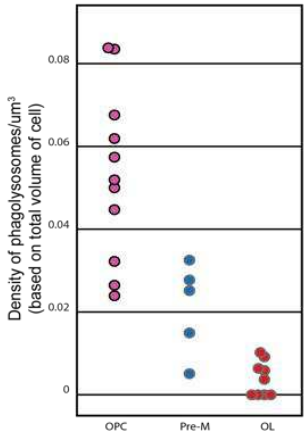

Extended data Figure 3. Premyelinating cells, myelinating oligodendrocyte and phagolysosome densities.

a, 3D rendering of a premyelinating oligodendrocyte (OL) in P49 dataset shows a branch aligned with an axon (white)(boxed area). Scale bar, $20 \mu \mathrm{m}$. b. Higher magnification view of boxed area shows myelin (orange) and axon (white). Scale bar, $1.5 \mu \mathrm{m}$. c, Ultrathin section view shows the uncompacted myelin(orange)(arrows) on the outside of the compact myelin (black). Scale bar, $750 \mathrm{~nm}$. d, Mature myelinating oligodendrocyte (OL) has thin branches that are aligned with colored myelinated axons. Scale bar, $10 \mu \mathrm{m}$. e-g, Plots comparing densities of phagolysosome distribution in OPCs, Premyelinating cells and mature oligodendrocytes. 


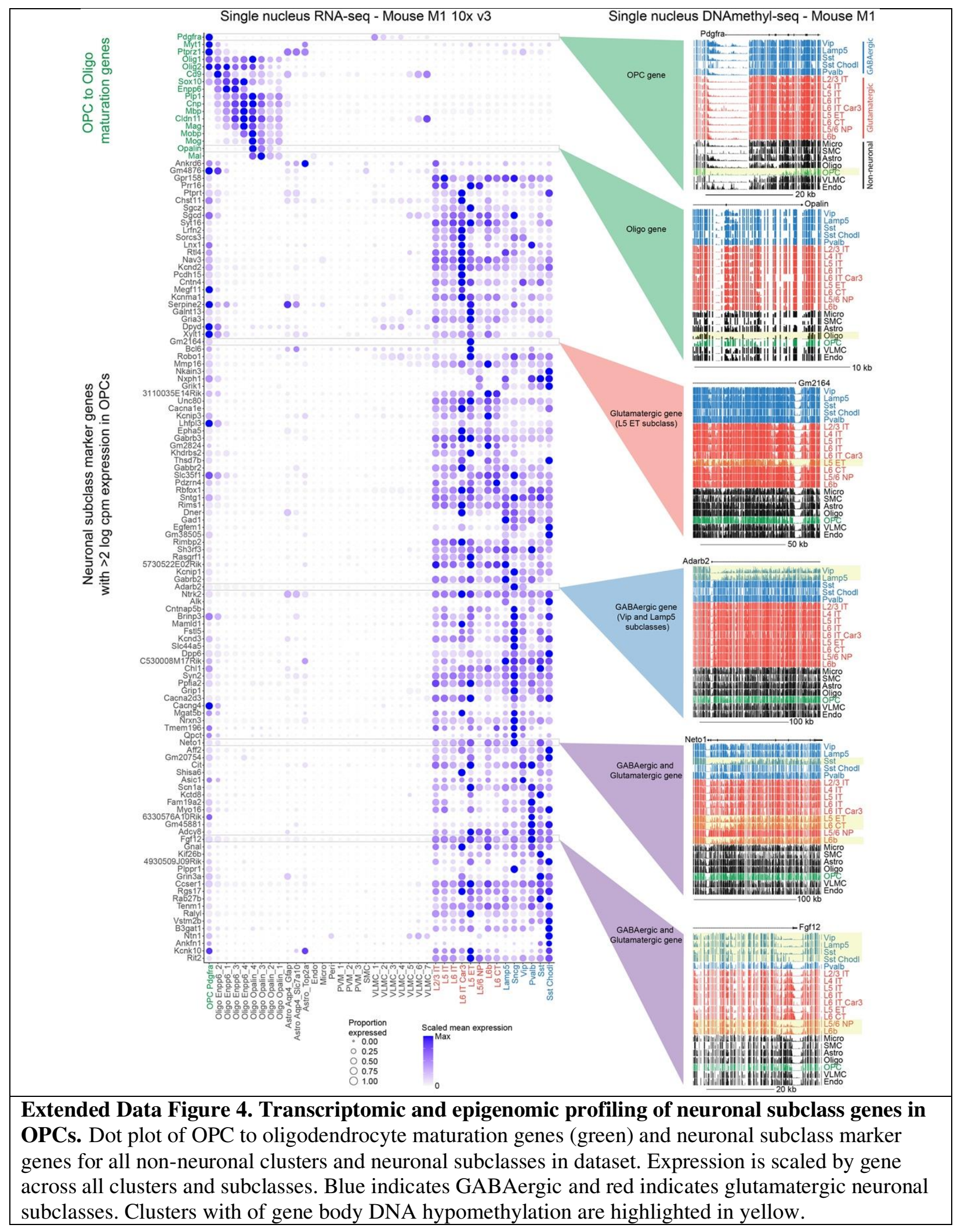



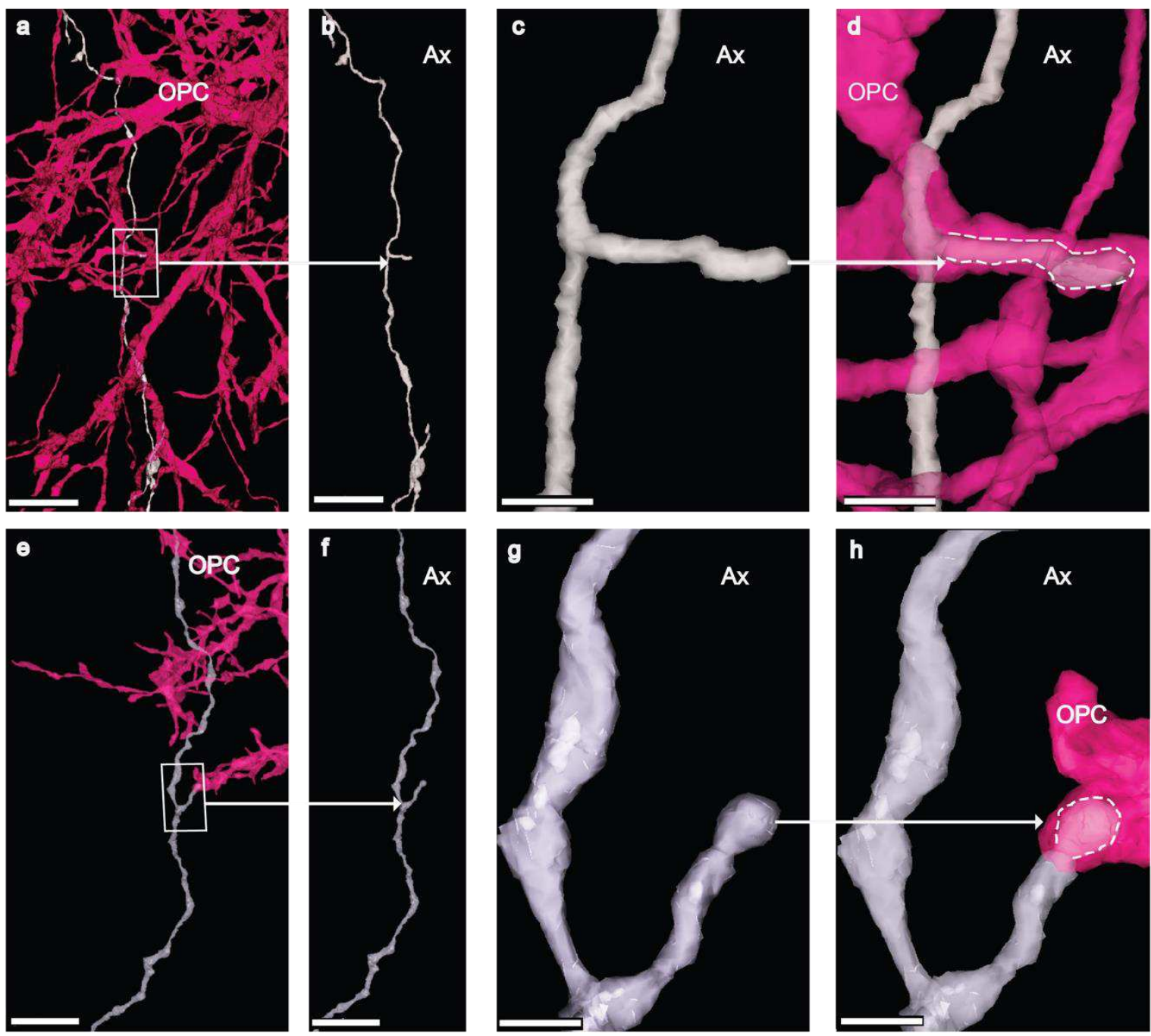

Extended Figure 5. OPCs ingest collateral branches.

a, OPC (pink) contacts an excitatory axon (gray). Boxed area shows site of ingestion. Scale bar, $5 \mu \mathrm{m}$. b, $3 \mathrm{D}$ rendering of the excitatory axon (gray). Arrow points to collateral branch in boxed area in (a). Scale bar, $5 \mu \mathrm{m}$. c, Higher magnification of collateral branch. White arrow points to ingested branch in (d). Scale bar, $750 \mathrm{~nm}$. d, 3D rendering of excitatory axon (gray) ingested within the branch of an OPC (pink). Dotted line indicates the outline of ingested collateral branch. Scale bar, $750 \mathrm{~nm}$. e, OPC (pink) contacts inhibitory axon (gray). Boxed area shows site of ingestion. Scale bar, $5 \mu \mathrm{m}$. f, 3D rendering of inhibitory axon (gray). Arrow points to collateral branch in boxed area in (e). Scale bar, $5 \mu \mathrm{m}$. $\mathbf{g}$, Higher magnification of collateral branch. White arrow points to ingested branch in (e). Scale bar, $750 \mathrm{~nm}$. h, 3D rendering of inhibitory axon (grey) ingested within the branch of an OPC (pink). Dotted line indicates the outline of ingested collateral branch. Scale bar, $750 \mathrm{~nm}$. Also see supplementary video 3 . 


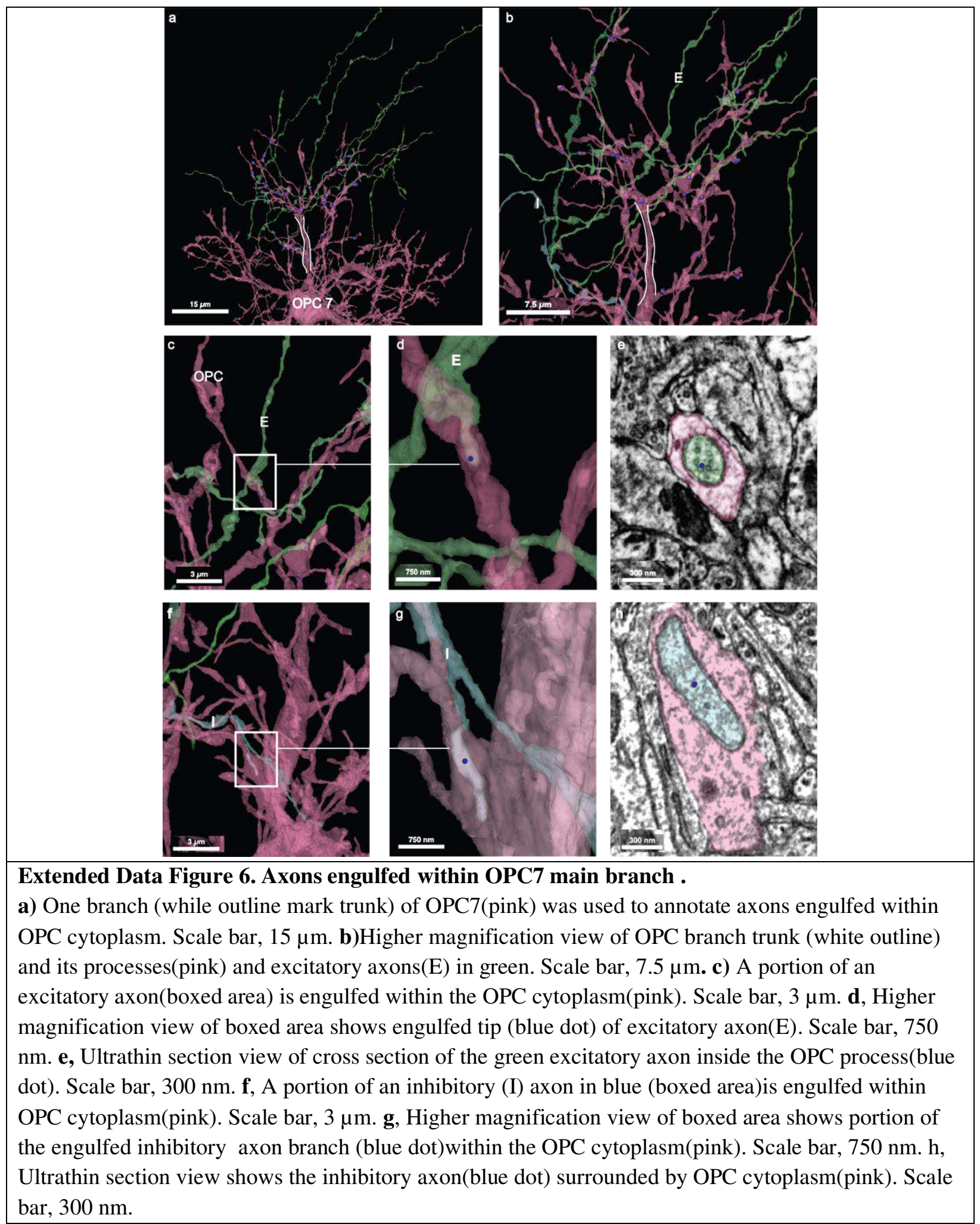


Conceptualization J.B., D.E.B., N.L. J., T.E. B., N.M.C; Software (Alignment) G.M., F.C., R.T., T.M.,W.M.S and W.W.; Software (Analysis infrastructure) C.S.J., C.S.M., F.C and S.D., S.S..; Software (Data interaction and viewing) M.C., N.K. and W.M.S.; Software (Cloud data storage) W.M.S.; Software Proofreading System) J.Zu., N.K. and S.D.; Formal Analysis A.N., B.H.,

\section{Acknowledgments}

We thank John Philips, Sill Coulter and the Program Management team at the AIBS for their guidance for project strategy and operations. We thank Hongkui Zeng, Christof Koch and Allan Jones for their support and leadership. We thank Andreas Tolias, Jacob Reimer and their teams at the Baylor College of Medicine for providing the mice used for electron microscopy. We thank the Manufacturing and Processing Engineering team at the AIBS for their help in implementing the EM imaging and sectioning pipeline. We thank Rob Young and the Technology team at the AIBS for their work in the imaging processing of the EM data. We thank Brian Youngstrom, Stuart Kendrick and the Allen Institute IT team for support with infrastructure, data management and data transfer. We thank the Facilities, Finance, and Legal teams at the AIBS for their support on the MICrONS contract. We thank Stephan Saalfeld for help with the parameters for 2D stitching and rough alignment of the dataset. We would like to thank the "Connectomics at Google" team for developing Neuroglancer and computational resource donations. We also would like to thank Amazon and Intel for their assistance. We thank S. Koolman, M. Moore, S. Morejohn, B. Silverman, K. Willie, and R. Willie for their image analyses, Garrett McGrath for computer system administration, and May Husseini and Larry and Janet Jackel for project administration. We thank Kristina Micheva and Stephen J Smith for advice and feedback. We thank Profs. Joseph Ayers, Erin Cram and James Monaghan for guidance, support and feedback. Supported by the Intelligence Advanced Research Projects Activity (IARPA) via Department of Interior/ Interior Business Center (DoI/IBC) contract numbers D16PC00003, D16PC00004, and D16PC0005. The U.S. Government is authorized to reproduce and distribute reprints for Governmental purposes notwithstanding any copyright annotation thereon. Disclaimer: The views and conclusions contained herein are those of the authors and should not be interpreted as necessarily representing the official policies or endorsements, either expressed or implied, of IARPA, DoI/IBC, or the U.S. Government. HSS also acknowledges support from NIH/NINDS U19 NS104648, and research reported in this publication was supported by the National Institute of Mental Health of the National Institutes of Health under Award Number U19MH114824. 
855 ARO W911NF-12-1-0594, NIH/NEI R01 EY027036, NIH/NIMH U01 MH114824, NIH/NINDS 856 R01NS104926, NIH/NIMH RF1MH117815. NIH/NIA R01AG072305 (DB), the Dr. Miriam and 857 Sheldon G Adelson Medical Research Foundation (DB), and the Mathers Foundation. The content 858 is solely the responsibility of the authors and does not necessarily represent the official views of 859 the National Institutes of Health.

860 We thank the Allen Institute for Brain Science founder, Paul G. Allen, for his vision, 861 encouragement and support.

862

863 


\section{Supplementary Files}

This is a list of supplementary files associated with this preprint. Click to download.

- mgcliptest5.mp4

- UsingNeuroglancertoAnalyseOPCsvideo2.mp4

- jbsupplmentalvideov1.mp4 